\title{
CATEGORIES WITH PROJECTIVE FUNCTORS
}

\author{
Oleksandr Khomenko
}

Dissertation zur Erlangung des Doktorgrades der Fakultät für Mathematik und Physik der Albert-Ludwigs-Universität Freiburg im Breisgau

November 2003 
Dekan: Prof. Dr. R. Schneider

Erster Referent: Prof. Dr. W. Soergel

Zweiter Referent: Prof. Dr. H. H. Andersen

Datum der Promotion: 13 Februar 2004 


\section{ACKNOWLEDGEMENTS}

First of all I thank my scientific advisor Wolfgang Soergel for his friendly supervision, motivational discussions, many useful remarks, patience and for sharing his deep ideas and insights on mathematics. I thank Volodymyr Mazorchuk for useful and friendly discussions, suggestions, ideas, hospitality during my visits to Uppsala and for careful reading of this manuscript. I thank my colleagues Bijan Afshordel, Peter Fiebig, Steen Ryom-Hansen, Gerald Höhn, Viktor Levandovskyy, Vladimir Matveev, Olaf Schnürer, Vsevolod Shevchishin and Catharina Stroppel for creating friendly and motivating working atmosphere. Especially I would like to thank my wife Nataliya Koval for emotional support.

I Acknowledge the financial support of Freiburg University, Uppsala University, TMR program "Algebraic Lie Representations", and DFG project "Projektive Funktoren und Hecke Algebra". 



\title{
CATEGORIES WITH PROJECTIVE FUNCTORS
}

\author{
O.KHOMENKO
}

\section{CONTENTs}

1. Introduction 1

2. Full Projective Functors 5

3. Preliminaries from Representation Theory 13

4. Some subcategories of $\mathfrak{g}$-mod $\quad 15$

5. Category $\mathcal{O} \quad 19$

5.1. Projective functors on $\mathcal{O} \quad 19$

5.2. Zuckerman functors 20

5.3. Joseph's version of completion functor 20

5.4. Coapproximation and functor $\mathbb{V} \quad 20$

5.5. Arkhipov's functor and Deodhar's version of completion functor 21

5.6. Relations between functors on $\mathcal{O} 24$

5.7. Parabolic category $\mathcal{O} 25$

6. Categorical Realization of Arkhipov's and Joseph's Functors 26

7. Harish-Chandra bimodules 30

8. Kostant's Problem 33

9. Structure of Induced Modules 36

References 43

\section{INTRODUCTION}

In the representation theory of objects associated to Dynkin diagrams (e.g. Lie algebras, Lie groups, Quantum groups) one often has translation functors. These functors encode certain symmetry of the representation category. In this paper we axiomatize some properties of these functors in the definition of a category with full projective functors (see Definition 1) in order to understand better the common features of such categories. A category $\mathcal{A}$ with full projective functors, is an abelian category with a distinguished object, called dominant object (an analog of the dominant Verma module in $\mathcal{O}$ ), and a collection of endofunctors subject to the axioms from Definition 1. 
This approach leads to a better understanding of right exact functors which sufficiently naturally commute with projective functors (see Definition 2 for a more precise explanation of "sufficiently natural"). One of the first attempts of axiomatic characterization of some functors on category $\mathcal{O}$ goes back to A. Joseph [J83], where he investigates a version of Enright's completion functor, in particular he proves that these functors satisfy the braid relations. Later H. H. Andersen, C. Stroppel in $[\mathrm{AnS}]$ established some properties of Arkhipov's functor and V. Mazorchuk and the author in [KM1] established some relations between different versions of Enright's functor, Arkhipov's functor (introduced in $[\mathrm{Ar}]$ ) and certain categorically defined functors. One of the main results of the present paper is the following theorem

Theorem 1. Right exact additive functors from a category $\mathcal{A}$ with full projective functors to an abelian category $\mathcal{B}$ which naturally commute with projective functors are determined up to an isomorphism of functors by their values on the dominant object.

Moreover one can provide a classification of such functors if one describes the objects of $\mathcal{B}$ which can appear as images of the dominant object in $\mathcal{A}$. Such objects will be called quasi-dominant objects. Definition 3 and Theorem 5 provide a description of these objects in terms of projective functors. Another important result of the paper is

Theorem 2. Every morphism between the values on the dominant object of right-exact additive functors which naturally commute with projective functors can be naturally lifted to a morphism of functors.

Unfortunately the correspondence in the above theorem is, in general, only injective. In other words one looses some information about morphisms of functors when evaluating them on the dominant object. An attempt to explain the nature of this phenomena is made at the end of Section 2. Clearly, the dual notions can be developed to deal with left exact additive functors. The above ideas can also be generalized to work in derived categories with full projective functors.

Going back to the original motivation, one can apply these results to Joseph's and Deodhar's versions of completion functor, Arkhipov's functor, Zuckerman's functor, slightly modified Soergel's combinatorial functor $\mathbb{V}$ and various categorically defined functors. After proving or citing the fact that these functors naturally commute with projective functors on the category $\mathcal{O}$ for semisimple finite dimensional complex Lie algebra $\mathfrak{g}$, one easily gets the braid relations for Joseph's completion functors and Arkhipov's functors, a description of Arkhipov's functor as the twist of Joseph's completion functor by duality, the 
isomorphism of the (slight modification of) functor $\mathbb{V}$ and the square of Arkhipov's functor, a description of Zuckerman's functor as left derived of Arkhipov's functor and various categorical realizations of above functors. These relations are proved just by computing corresponding functors on the dominant Verma module. Some of the above relations are already known (see [An, AnS, AnL, Ar, J83, KM1]) some of them seem to be new.

A priori the definition of a category with full projective functors depends on the choice of the dominant object. However in many cases one gets equivalent constructions. More precisely we have

Theorem 3. Let $\left(\mathcal{A}, M,\left\{\mathcal{P}_{i}, i \in \mathcal{I}^{\prime}\right\}\right)$ and $\left(\mathcal{A}^{\prime}, M^{\prime},\left\{\mathcal{P}_{i}^{\prime}, i \in \mathcal{I}^{\prime}\right\}\right)$ be two categories with full projective functors such that

(1) there exist an additive functor $\mathfrak{F}: \underline{\operatorname{Hom}}(\mathcal{A}, \mathcal{A}) \rightarrow \underline{\operatorname{Hom}}\left(\mathcal{A}^{\prime}, \mathcal{A}^{\prime}\right)$ which induces an equivalence of monoidal categories on categories of projective functors and maps projective functors on $\mathcal{A}$ to right exact additive functors on $\mathcal{A}^{\prime}$

(2) for all $i, j \in \mathcal{I}$ and $\mathfrak{t} \in \operatorname{Hom}_{F u n c}\left(\mathcal{P}_{i}, \mathcal{P}_{j}\right)$ one has $\mathfrak{t}_{M}=0$ if and only if $\mathfrak{F}(\mathfrak{t})_{M^{\prime}}=0$.

Then $\mathcal{A}$ and $\mathcal{A}^{\prime}$ are equivalent.

Using this theorem one can establish for example the equivalences of various categories of modules which are presentable by certain class of projective modules. In this way one can deduce some properties of arbitrary $\mathfrak{g}$-modules from analogous properties of modules in $\mathcal{O}$. As an application we obtain an answer to Kostant's question (a description of the algebra of adjoint finite endomorphisms) for simple modules with a minimal annihilator.

Theorem 4. Let $M$ be a simple $\mathfrak{g}$-module with minimal annihilator. Then the algebra of adjoint finite endomorphisms of $M$ is a free $U(\mathfrak{g}) / \operatorname{Ann}_{U(\mathfrak{g})} M$-module of finite rank.

Moreover the rank of the module in the theorem can be described explicitly (see Section 8). The solution of this problem is well known for Verma modules, and in [Za] the problem was solved for Whittaker modules. We have to remark that the canonical morphism from $U(\mathfrak{g})$ to the algebra of adjoint finite morphisms of a simple module given by multiplication is not surjective in general, (even for modules in category $\mathcal{O})$ see [GJ, S89] for examples.

Another application of the technique is a description of the structure of parabolicaly induced modules. This problem was solved by D. Miličić and W. Soergel in [MS] for Whittaker modules. Their ideas was adopted to work in the case of arbitrary simple module $V$ with 
minimal annihilator by V. Mazorchuk and the author in [KM2]. However, if for some finite dimensional module $E$ the length of $E \otimes V$ does not equal to the dimension of $E$ one may loose some information about subquotients of induced module. The proofs presented here are slight modifications of those in [KM2]. The central idea of [MS] and [KM2] is that the (rough) structure of the module which is parabolicaly induced from a simple module with minimal annihilator depends only on its central character.

The methods used in the investigation of Kostant's problem and of the structure of induced modules can be adopted to work under some restrictions also for simple modules whose annihilator is an induced ideal. A more detailed discussion of the difficulties appearing in this case can be found at the end of Section 8 .

The discussed technique can also be applied to the category of HarishChandra bimodules with (generalized) central character from the right hand side (the last however need to be modified a bit). In this case one obtains two different structures of a category with full projective functors on it coming from the tensoring with finite dimensional modules from the left hand side and from the right hand side. The second one provides another structure of category with full projective functors on $\mathcal{O}_{\lambda}$, which can be used to deal with translation functors, ("classical") projective functors, shuffling functors and Zuckerman functors. In particular, one can reprove the result of Bernstein and Gelfand that ("classical") projective functors on $\mathcal{O}$ are determined by their values on the dominant Verma module.

Let us describe the structure of the paper. In Section 2 we develop the main abstract constructions. In particular we present the definition of a category with full projective functors and prove the main results concerning description of right (left) exact additive functors which naturally commute with projective functors. After fixing notations and recalling some basic results from the representation theory in Section 3 we construct an example of a category with full projective functors and some functors on it in Section 4. The structure of a category with full projective functors on $\mathcal{O}$ is discussed in more details in Section 5. Here we also show that many "classical" functors on $\mathcal{O}$ naturally commute with projective functors. Moreover it appears that there are not too much "essentially" different functors among them. In this section we also explain how the parabolic category $\mathcal{O}^{\mathfrak{p}}$ can be viewed as a category with full projective functors. We have to admit that the description of the homomorphism space between two projective functors on parabolic category $\mathcal{O}$ seems to be rather difficult problem. In Section 6 we 
present a categorical realization of Joseph's version of Enright's completion functors and of Arkhipov's functor. This result was obtained by V. Mazorchuk and the author in [KM1]. In Section 7 we apply the abstract nonsense to a certain completion of the category of HarishChandra bimodules. The Kostant's problem is attacked in Section 8 and the structure of induced modules is investigated in Section 9.

\section{Full Projective Functors}

Let $\mathcal{A}, \mathcal{B}$ and $\mathcal{C}$ be abelian categories. $\operatorname{By} \operatorname{Hom}(\mathcal{A}, \mathcal{B})$ we denote the category of functors from $\mathcal{A}$ to $\mathcal{B}$. The objects of $\underline{\operatorname{Hom}}(\mathcal{A}, \mathcal{B})$ are all functors from $\mathcal{A}$ to $\mathcal{B}$ and the morphisms are natural transformations of functors. The category $\operatorname{Hom}(\mathcal{A}, \mathcal{B})$ is an additive strict monoidal category with respect to composition ${ }_{-}{ }_{-}$of functors. Let $\mathbf{I d}_{\mathcal{A}}$ denote the identity functor on $\mathcal{A}$. For two objects $F, G \in \operatorname{Ob}(\underline{\operatorname{Hom}}(\mathcal{A}, \mathcal{B}))$ by $\operatorname{Hom}_{F u n c}(F, G)$ we denote the set of natural transformations (morphisms of functors) from $F$ to $G$. Let $H, H^{\prime} \in \operatorname{Ob}(\underline{\operatorname{Hom}}(\mathcal{B}, \mathcal{B}))$ and $\mathfrak{H} \in$ $\operatorname{Hom}_{\text {Func }}\left(H, H^{\prime}\right)$. For a morphism $\mathfrak{f} \in \operatorname{Hom}_{F u n c}(F, G)$ and $K \in \operatorname{Ob}(\mathcal{A})$ by $\mathfrak{f}_{K}: F(K) \rightarrow G(K)$ we denote the evaluation of $\mathfrak{f}$ on $K$, by $H \circ \mathfrak{f}$ we denote the natural transformation from $H \circ F$ to $H \circ G$ defined by $(H \circ \mathfrak{f})_{N}=H\left(\mathfrak{f}_{N}\right)$ and by $\mathfrak{H} \circ F \in \operatorname{Hom}_{F u n c}\left(H \circ F, H^{\prime} \circ F\right)$ we denote the natural transformation defined by $(\mathfrak{H} \circ F)_{N}=\mathfrak{H}_{F(N)}$ for $N \in \operatorname{Ob}(\mathcal{A})$.

Definition 1. A category with full projective functors is an abelian category $\mathcal{A}$ together with an object $M \in \mathrm{Ob}(\mathcal{A})$ (called dominant object) and a collection of right exact and additive endofunctors $\mathcal{P}_{i}, i \in \mathcal{I}$ (called projective functors), closed under taking direct sums and compositions of functors such that

(PF1) The identity functor $\mathbf{I d}_{\mathcal{A}}$ is a projective functor.

(PF2) For every $i \in \mathcal{I}$ the object $\mathcal{P}_{i}(M)$ is projective in $\mathcal{A}$.

(PF3) For every $N \in \mathrm{Ob}(\mathcal{A})$ there exist a projective functor $\mathcal{P}^{N}$ and an epimorphism $f_{N}: \mathcal{P}^{N}(M) \rightarrow N$.

(PF4) For all $i, j \in \mathcal{I}$ the evaluation map

$$
\operatorname{ev}_{M}: \operatorname{Hom}_{F u n c}\left(\mathcal{P}_{i}, \mathcal{P}_{j}\right) \rightarrow \operatorname{Hom}_{\mathcal{A}}\left(\mathcal{P}_{i}(M), \mathcal{P}_{j}(M)\right)
$$

is surjective.

The above definition immediately implies that $M$ is projective and any projective module $P$ is a direct summand of $\mathcal{P}^{P}(M)$. Let $\mathcal{Q}$ be a projective functor, then $\mathcal{Q}(P)$ is a direct summand of $\mathcal{Q} \circ \mathcal{P}^{P}(M)$, thus it is projective.

Let $\overline{\mathcal{A}}=\left(\mathcal{A}, M,\left\{\mathcal{P}_{i} \mid i \in \mathcal{I}\right\}\right)$ be a category with full projective functors. By $\mathcal{E}_{\overline{\mathcal{A}}}$ we will denote the category of projective functors of $\overline{\mathcal{A}}$. 
By definition, the objects of $\mathcal{E}_{\overline{\mathcal{A}}}$ are the functors $\mathcal{P}_{i}, i \in \mathcal{I}$ and morphisms are the natural transformations of these functors. For any object $N \in \operatorname{Ob}(\mathcal{A})$ the evaluation on $N$ defines a functor from $\mathcal{E}_{\overline{\mathcal{A}}}$ to $\mathcal{A}$, which we denote by $\operatorname{ev}_{N}$. By construction $\operatorname{ev}_{N}\left(\mathcal{P}_{i}\right)=\mathcal{P}_{i}(N)$ and for any $\mathfrak{t} \in \operatorname{Hom}_{\text {Func }}\left(\mathcal{P}_{i}, \mathcal{P}_{j}\right)$ holds ev ${ }_{N}(\mathfrak{t})=\mathfrak{t}_{N}$. The Axiom (PF4) implies that the functor $\mathrm{ev}_{M}$ is full.

A functor $\mathfrak{F}: \mathcal{E}_{\overline{\mathcal{A}}} \rightarrow \underline{\operatorname{Hom}}(\mathcal{B}, \mathcal{B})$ is called admissible if it is additive, $\mathfrak{F}\left(\mathbf{I d}_{\mathcal{A}}\right)=\operatorname{Id}_{\mathcal{B}}, \mathfrak{F}\left(\widehat{\left.\mathcal{P}_{i}\right)}\right.$ is right exact, additive and $\mathfrak{F}\left(\mathcal{P}_{i} \circ \mathcal{P}_{j}\right)=$ $\mathfrak{F}\left(\mathcal{P}_{i}\right) \circ \mathfrak{F}\left(\mathcal{P}_{j}\right)$ for all $i, j \in \mathcal{I}$. For example, if $\mathcal{A}=\mathcal{B}$ then the identity functor on $\operatorname{\operatorname {Hom}}(\mathcal{A}, \mathcal{A})$ is always admissible. Fix a category with full projective functors $\overline{\mathcal{A}}=\left(\mathcal{A}, M,\left\{\mathcal{P}_{i} \mid i \in \mathcal{I}\right\}\right)$, an abelian category $\mathcal{B}$ and an admissible functor $\mathfrak{F}: \mathcal{E}_{\overline{\mathcal{A}}} \rightarrow \operatorname{Hom}(\mathcal{B}, \mathcal{B})$ for the rest of this section.

Definition 2. A functor $G: \mathcal{A} \rightarrow \mathcal{B}$ naturally $\mathfrak{F}$-commutes with projective functors if there exist a collection of isomorphisms of functors $\mathbf{C}_{i}^{G}: \mathfrak{F}\left(\mathcal{P}_{i}\right) \circ G \rightarrow G \circ \mathcal{P}_{i}, i \in \mathcal{I}$ such that for all $i, j \in \mathcal{I}$ and $\mathfrak{t} \in \operatorname{Hom}_{\text {Func }}\left(\mathcal{P}_{i}, \mathcal{P}_{j}\right)$ the following diagram commutes:

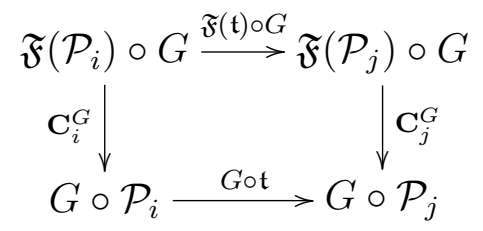

If the functor $\mathfrak{F}$ is clear from the context we will sometimes omit it. Every morphism between values on the dominant object $M$ of right exact additive functors which naturally commute with projective functors comes from a natural transformation. More precisely we have

Proposition 1. Let $F$ and $G$ be two right exact additive functors from $\mathcal{A}$ to $\mathcal{B}$ which naturally $\mathfrak{F}$-commute with projective functors, then for every morphism $\phi: F(M) \rightarrow G(M)$ there exist a natural (with respect to the composition of morphisms) lift of $\phi$ to a morphism $\mathfrak{f}^{\phi}: F \rightarrow G$ of functors such that $\mathfrak{f}_{M}^{\phi}=\phi$. Moreover, $\phi$ is an isomorphism if and only if $\mathfrak{f}^{\phi}$ is.

Proof. Given $\phi: F(M) \rightarrow G(M)$ let us construct $\mathfrak{f}^{\phi}$ as follows. For all $i \in \mathcal{I}$ the morphism $\mathfrak{f}_{\mathcal{P}_{i}(M)}^{\phi}: F\left(\mathcal{P}_{i}(M)\right) \rightarrow G\left(\mathcal{P}_{i}(M)\right)$ is defined to be equal to $\left(\mathbf{C}_{i}^{G}\right)_{M} \circ \mathfrak{F}\left(\mathcal{P}_{i}\right)(\phi) \circ\left(\mathbf{C}_{i}^{F}\right)_{M}^{-1}$. Note that if $\phi$ is an isomorphism, then $\mathfrak{f}_{\mathcal{P}_{i}(M)}^{\phi}$ are also isomorphisms. 
Let $i, j \in \mathcal{I}$ and $t \in \operatorname{Hom}_{\text {Func }}\left(\mathcal{P}_{i}, \mathcal{P}_{j}\right)$. The top face of the following diagram is commutative by the definition of a natural transformation. (1)

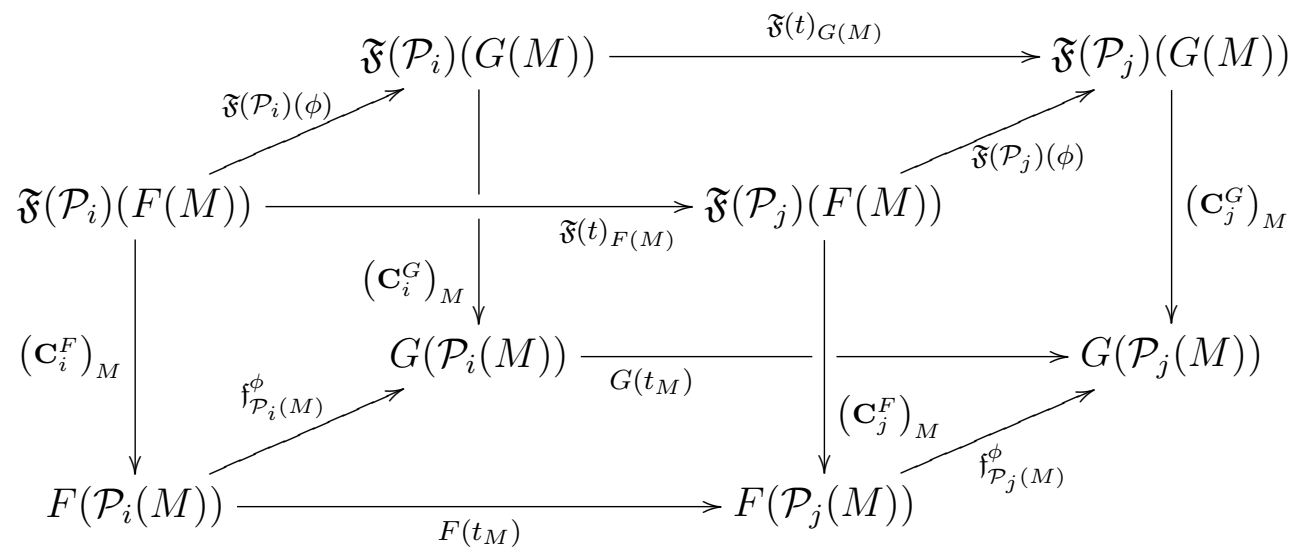

The commutativity of front and back faces is given by Definition 2, and right and left faces commute by construction of $\mathfrak{f}^{\phi}$. Since vertical arrows are isomorphisms one gets the commutativity of the bottom face.

By the definition of a category with full projective functors, for every object $N \in \mathrm{Ob}(A)$ there exist projective functors $\mathcal{P}_{1}$ and $\mathcal{P}_{2}$ on $\mathcal{A}$ and $\mathfrak{t} \in \operatorname{Hom}_{\text {Func }}\left(\mathcal{P}_{1}, \mathcal{P}_{2}\right)$ such that $N$ fits into the following exact sequence

$$
\mathcal{P}_{1}(M) \stackrel{\mathfrak{t}_{M}}{\longrightarrow} \mathcal{P}_{2}(M) \stackrel{p}{\longrightarrow} N \longrightarrow 0 .
$$

The commutativity of the Diagram (1) implies the commutativity of the left square of the following diagram with exact rows

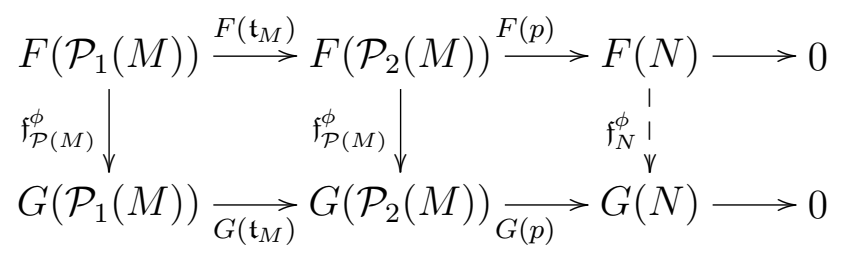

We define $\mathfrak{f}_{N}^{\phi}$ to be the morphism making this diagram commutative. By standard arguments $\mathfrak{f}_{N}^{\phi}$ does not depend on the choice of $\mathcal{P}_{1}, \mathcal{P}_{2}$ and $\mathfrak{t}$. Note that if $\phi$ is an isomorphism, then $\mathfrak{f}_{N}^{\phi}$ is also an isomorphism.

Let $N^{1}, N^{2} \in \operatorname{Ob}(\mathcal{A})$ and $g \in \operatorname{Hom}_{\mathcal{B}}\left(N^{1}, N^{2}\right)$. By standard arguments $g$ can be lifted to a morphism between projective presentations of $N^{1}$ and $N^{2}$. By the definition of a category with full projective functors, it can be lifted to morphisms between corresponding projective functors. All together we get: there exist projective functors $\mathcal{P}_{i}^{j}$, $i, j=1,2$ on $\mathcal{A}$ and appropriate natural transformations $\mathfrak{t}^{1}, \mathfrak{t}^{2}, \mathfrak{g}^{1}, \mathfrak{g}^{2}$ 
of these functors such that the following diagram commutes.

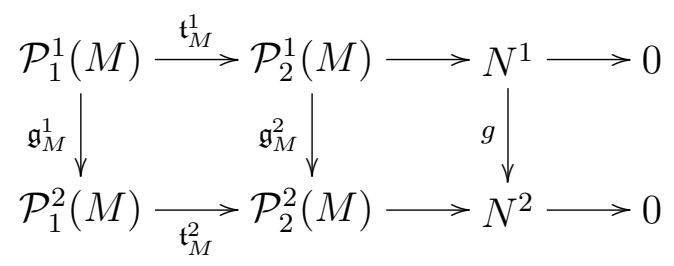

The commutativity of (1) implies the commutativity of the following four diagrams (here $i=1,2$ )
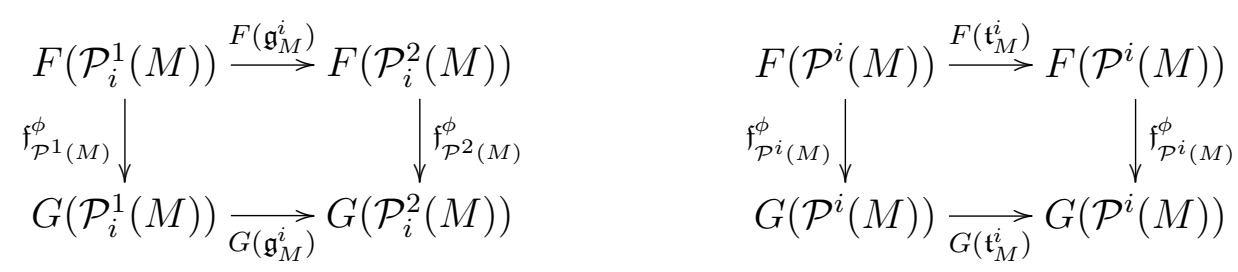

Thus, applying functors $F$ and $G$ to Diagram (2) and "gluing" the result with the four diagrams above, we get the commutativity of

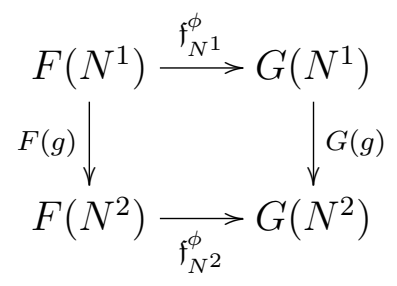

This proves that the collection $\mathfrak{f}_{N}^{\phi}, N \in \mathrm{Ob}(\mathcal{A})$ defines a natural transformation $\mathfrak{f}^{\phi}$ from $F$ to $G$. The equality $\mathfrak{f}_{M}^{\phi}=\phi$ can easily be obtained if one takes $0 \rightarrow M \cong M \rightarrow 0$ as a projective resolution of $M$ and considers the following commutative diagram:

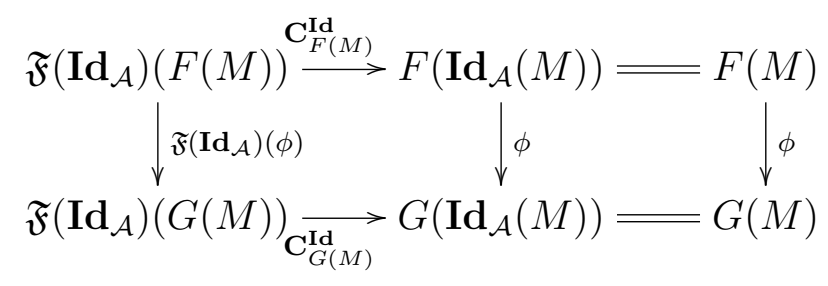

where $\mathbf{C}_{F(M)}^{\mathbf{I d}}$ and $\mathbf{C}_{G(M)}^{\mathbf{I d}}$ are the isomorphisms given by Definition 2 for the identity functor.

The naturality of such lift with respect to composition of morphisms follows from the construction. 
Rather unexpected is the fact that in general not all objects of $\mathcal{B}$ can appear as the image of the dominant object under a right exact additive functor which naturally $\mathfrak{F}$-commutes with projective functors.

Definition 3. We say that an object $N$ of $\mathcal{B}$ is $\mathfrak{F}$-quasi-dominant if for all $i, j \in \mathcal{I}$ and for all $\mathfrak{t} \in \operatorname{Hom}_{F u n c}\left(\mathcal{P}_{i}, \mathcal{P}_{j}\right)$ the equality $\mathfrak{t}_{M}=0$ implies $\mathfrak{F}(\mathfrak{t})_{N}=0$.

Proposition 2. Let $N$ be an $\mathfrak{F}$-quasi-dominant object in $\mathcal{B}$. Then there exist a unique up to isomorphism right exact additive functor $G \in \operatorname{Ob}(\underline{\operatorname{Hom}}(\mathcal{A}, \mathcal{B}))$ such that $G$ naturally $\mathfrak{F}$-commutes with projective functors and $G(M) \cong N$.

Proof. The uniqueness immediately follows from Proposition 1. Let us prove the existence. By the definition of a category with full projective functors it is enough to define $G$ on $\operatorname{coker}\left(\mathfrak{t}_{M}\right)$ where $\mathfrak{t}: \mathcal{P}_{1} \rightarrow \mathcal{P}_{2}$ is a natural transformation between two projective functors $\mathcal{P}_{1}$ and $\mathcal{P}_{2}$. Let us define $G\left(\mathcal{P}_{i}(M)\right)=\mathfrak{F}\left(\mathcal{P}_{i}\right)(N)$ and $G\left(\operatorname{coker}\left(\mathfrak{t}_{M}\right)\right)=\operatorname{coker}\left(\mathfrak{F}(\mathfrak{t})_{N}\right)$.

Suppose we are given two projective functors, $\tilde{\mathcal{P}}_{1}, \tilde{\mathcal{P}}_{2}$, a natural transformation $\tilde{\mathfrak{t}} \in \operatorname{Hom}_{F u n c}\left(\tilde{\mathcal{P}}_{1}, \tilde{\mathcal{P}}_{2}\right)$ and $g \in \operatorname{Hom}_{\mathcal{A}}\left(\operatorname{coker}\left(\mathfrak{t}_{M}\right)\right.$, $\left.\operatorname{coker}\left(\tilde{\mathfrak{t}}_{M}\right)\right)$. There exist natural transformations $\mathfrak{g}^{i} \in \operatorname{Hom}_{\text {Func }}\left(\mathcal{P}_{i}, \tilde{\mathcal{P}}_{i}\right), i=1,2$ such that the following diagram with exact rows commutes

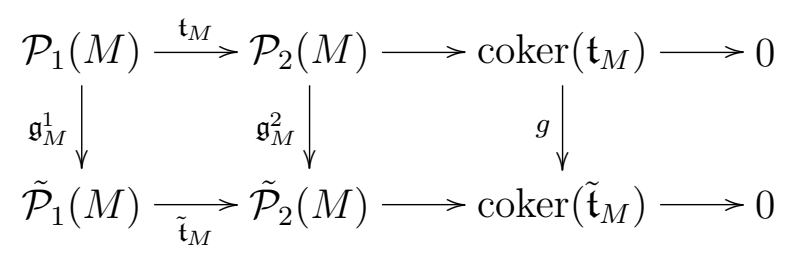

We define $G(g)$ to be the unique morphism making the following diagram with exact rows commute

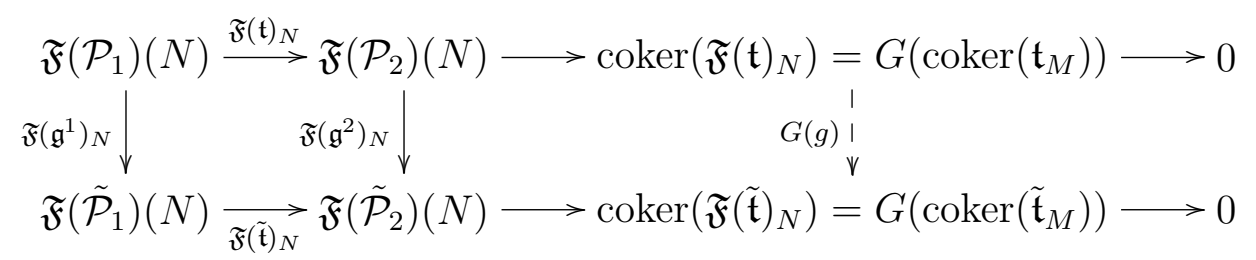

Let $\tilde{\mathfrak{g}}^{i} \in \operatorname{Hom}_{F u n c}\left(\mathcal{P}_{i}, \tilde{\mathcal{P}}_{i}\right)$ be such that $\tilde{\mathfrak{g}}_{M}^{i}-\mathfrak{g}_{M}^{i}=0$ for $i=1$, 2. Since $N$ is quasidominant and $\mathfrak{F}$ is additive, it follows that $\mathfrak{F}\left(\tilde{\mathfrak{g}}^{i}\right)_{N}-\mathfrak{F}\left(\mathfrak{g}^{i}\right)_{N}=$ 0 for $i=1,2$. So $G(g)$ does not depend on the lifts of morphisms $\mathfrak{g}_{M}^{i}, i=1,2$, to natural transformations of projective functors. The independence of $G(g)$ of the choice of the morphisms $\mathfrak{g}_{M}^{i}$ can be proved by standard diagram chaise. 
The equality $G\left(g \circ g^{\prime}\right)=G(g) \circ G\left(g^{\prime}\right)$ for suitable morphism $g^{\prime}$ and the fact that $G$ sends the identity morphism to the identity morphism are clear from the construction.

Let $\mathcal{Q}$ be a projective functor. One has the following presentation

$$
\mathcal{Q} \circ \mathcal{P}_{1}(M) \stackrel{\mathcal{Q}^{\circ} \mathfrak{t}_{M}}{\longrightarrow} \mathcal{Q} \circ \mathcal{P}_{2}(M) \longrightarrow \mathcal{Q}\left(\operatorname{coker}\left(\mathfrak{t}_{M}\right)\right) \longrightarrow 0
$$

of $\mathcal{Q}\left(\operatorname{coker}\left(\mathfrak{t}_{M}\right)\right)$. As far as $\mathcal{Q} \circ \mathcal{P}_{i}, i=1,2$ are projective functors we have by construction $G\left(\mathcal{Q} \circ \mathcal{P}_{i}(M)\right)=\mathfrak{F}\left(\mathcal{Q} \circ \mathcal{P}_{i}\right)(G(M))$ for $i=1,2$. This implies that $G$ naturally $\mathfrak{F}$-commutes with projective functors.

Now we are ready to present the classification of right exact additive functors which naturally $\mathfrak{F}$-commute with projective functors.

Theorem 5. The functor $G \mapsto G(M)$ from $\underline{\operatorname{Hom}}(\mathcal{A}, \mathcal{B})$ to $\mathcal{B}$ induces a bijection between the isomorphism classes of right exact additive functors from $\mathcal{A}$ to $\mathcal{B}$ which naturally $\mathfrak{F}$-commute with projective functors and the isomorphism classes of $\mathfrak{F}$-quasi-dominant objects of $\mathcal{B}$.

Proof. Let $G$ be a right exact additive functor from $\mathcal{A}$ to $\mathcal{B}$ which naturally $\mathfrak{F}$-commutes with projective functors. By the definition for all $i, j \in \mathcal{I}$ and $\mathfrak{t} \in \operatorname{Hom}_{\text {Func }}\left(\mathcal{P}_{i}, \mathcal{P}_{j}\right)$ one has

$$
(G \circ \mathfrak{t})_{M}=G\left(\mathfrak{t}_{M}\right)=\left(\mathbf{C}_{j}^{G}\right)_{M} \circ \mathfrak{F}(\mathfrak{t})_{G(M)} \circ\left(\mathbf{C}_{i}^{G}\right)_{M}^{-1} .
$$

In particular $\mathfrak{t}_{M}=0$ implies $\mathfrak{F}(\mathfrak{t})_{G(M)}=0$, thus the object $G(M)$ is $\mathfrak{F}$-quasidominant. Now the theorem easily follows from Proposition 1 and Proposition 2.

An immediate corollary from the results above enables one to construct equivalences of categories with full projective functors.

Corollary 1. Let $\overline{\mathcal{A}}=\left(\mathcal{A}, M,\left\{\mathcal{P}_{i}, i \in \mathcal{I}\right\}\right), \overline{\mathcal{A}}^{\prime}=\left(\mathcal{A}^{\prime}, M^{\prime},\left\{\mathcal{P}_{i}^{\prime}, i \in \mathcal{I}^{\prime}\right\}\right)$ be two categories with full projective functors such that

(1) there exist an admissible functor $\mathfrak{F}: \mathcal{E}_{\overline{\mathcal{A}}} \rightarrow \underline{\operatorname{Hom}}\left(\mathcal{A}^{\prime}, \mathcal{A}^{\prime}\right)$ which induces an equivalence on categories of projective functors

(2) for all $i, j \in \mathcal{I}$ and $\mathfrak{t} \in \operatorname{Hom}_{\text {Func }}\left(\mathcal{P}_{i}, \mathcal{P}_{j}\right)$ one has $\mathfrak{t}_{M}=0$ if and only if $\mathfrak{F}(\mathfrak{t})_{M^{\prime}}=0$.

Then $\mathcal{A}$ and $\mathcal{A}^{\prime}$ are equivalent.

Proof. Let $\mathbf{I} \mathbf{d}_{\mathcal{A}}$ and $\mathbf{I d}_{\mathcal{A}^{\prime}}$ be the identity functors on $\mathcal{A}$ and $\mathcal{A}^{\prime}$ respectively. Obviously they are right exact, additive and naturally $\mathfrak{F}^{-1} \circ \mathfrak{F}^{-}$ (resp $\mathfrak{F} \circ \mathfrak{F}^{-1}$-)commute with projective functors. By Proposition 2 there exist right exact additive functors $F: \mathcal{A} \rightarrow \mathcal{A}^{\prime}$ and $G: \mathcal{A}^{\prime} \rightarrow \mathcal{A}$ which naturally $\mathfrak{F}$-(resp. $\mathfrak{F}^{-1}$-) commute with projective functors such that $F(M)=M^{\prime}$ and $G\left(M^{\prime}\right)=M$. 
The functor $G \circ F$ is obviously right exact, additive and $\mathfrak{F}^{-1} \circ \mathfrak{F}^{-}$ commutes with projective functors, so by Proposition $1 G \circ F \cong \mathbf{I d}_{\mathcal{A}}$. Similarly $F \circ G \cong \mathbf{I d}_{\mathcal{A}^{\prime}}$, so $F$ and $G$ are mutually inverse equivalences of categories.

The ideas developed in this section can also be adopted to the investigation of functors on derived categories. Here we will present only one lemma which will be applied in the sequel.

Lemma 1. Let $F: \mathcal{A} \rightarrow \mathcal{B}$ be a right exact additive functor which naturally $\mathfrak{F}$-commute with projective functors, then, for all integer $i \geq 0$, the derived functors $\mathcal{L}_{i} F$ naturally $\mathfrak{F}$-commute with those exact projective functors whose images under $\mathfrak{F}$ are exact.

Proof. By Definition 1 any object $N$ of $\mathcal{A}$ has a projective resolution of type

$$
\cdots \rightarrow \mathcal{P}_{i}(M) \stackrel{f_{M}^{(i)}}{\rightarrow} \mathcal{P}_{i-1}(M) \rightarrow \cdots \rightarrow \mathcal{P}_{0}(M) \rightarrow N \rightarrow 0
$$

for some projective functors $\mathcal{P}_{i}, i \in \mathbb{N}$, and natural transformations $f^{(i)}: \mathcal{P}_{i} \rightarrow \mathcal{P}_{i-1}$. Let $\mathcal{Q}_{1}$ and $\mathcal{Q}_{2}$ be two exact projective functors and $g \in \operatorname{Hom}_{\text {Func }}\left(\mathcal{Q}_{1}, \mathcal{Q}_{2}\right)$. Choose a projective resolution

$\cdots \mathcal{Q}_{j} \circ \mathcal{P}_{i}(M) \stackrel{\mathcal{Q}_{j}\left(f_{M}^{(i)}\right)}{\longrightarrow} \mathcal{Q}_{j} \circ \mathcal{P}_{i-1}(M) \rightarrow \cdots \rightarrow \mathcal{Q}_{j} \circ \mathcal{P}_{0}(M) \rightarrow \mathcal{Q}_{j}(N) \rightarrow 0$

of $\mathcal{Q}_{j}(N), j=1,2$. By Definition 2 one gets the commutativity of the diagram

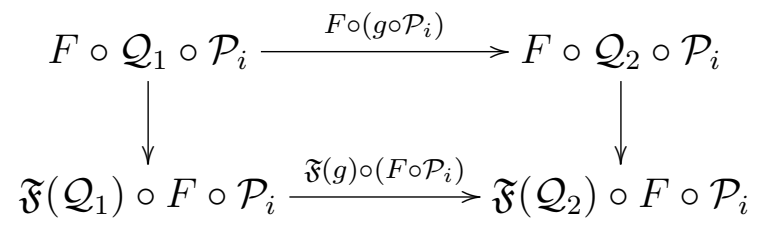

where the vertical arrows are isomorphisms of functors. On the other hand from the isomorphism of functors $F \circ \mathcal{Q}_{j} \cong \mathfrak{F}\left(\mathcal{Q}_{j}\right) \circ F$ we get the commutativity of

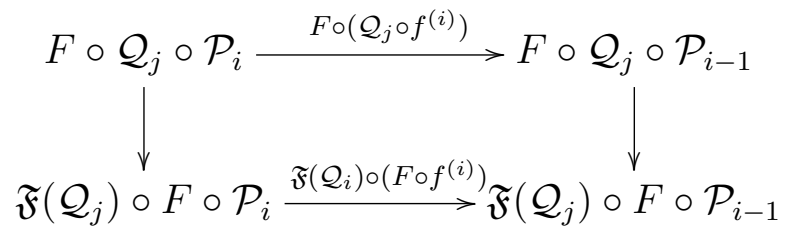

for $i \in \mathbb{N}, j=1,2$. The statement of the lemma can now be obtained by pasting these squares together and taking corresponding homologies. 
We have to remark that the correspondence from Theorem 5 induces a map from the space of natural transformations of right exact additive functors which naturally $\mathfrak{F}$-commute with projective functors to homomorphism space between their values on the dominant objects. This map is surjective by Proposition 1 but it is not injective in general. To understand the nature of this phenomena we have to explain the relation of Theorem 5 to Eilenberg-Watts theorem providing a classification of right continuous functors between two categories of modules (see [Ba, Theorem 2.3] for more details).

An object $P \in \mathrm{Ob}(\mathcal{A})$ is a projective generator of $\mathcal{A}$ if the functor $\operatorname{Hom}_{\mathcal{A}}\left(P,_{-}\right)$is exact and faithful. Let $\mathbb{K}$ be a field. Suppose that $\mathcal{A}$ is an $\mathbb{K}$-category with direct sums and there exist a projective functor $\mathcal{P}$ such that $\mathcal{P}(M)$ is a projective generator. Denote $A=\operatorname{Hom}_{\mathcal{A}}(\mathcal{P}(M), \mathcal{P}(M))$. The functor $\operatorname{Hom}_{\mathcal{A}}\left(\mathcal{P}(M),{ }_{-}\right)$defines an equivalence of categories from $\mathcal{A}$ to the category of finitely generated right $A$-modules (see [Ba, II.1]). Assume that there exist a $\mathbb{K}$-algebra $B$ such that $\mathcal{B} \cong \bmod -B$. The next theorem is a version of EilenbergWatts theorem.

Theorem 6. Let $A$ and $B$ be two $\mathbb{K}$-algebras. The correspondence $X \mapsto{ }_{-} \otimes_{A} X$ from $A-B$ bimodules to right continuous $\mathbb{K}$-functors from mod- $A$ to mod- $B$ induces a bijection on isomorphism classes. Moreover, given two $A-B$ bimodules $X$ and $Y$ the obvious map

$$
\operatorname{Hom}_{A-B}(X, Y) \rightarrow \operatorname{Hom}_{F u n c}\left(-\otimes_{A} X,{ }_{-} \otimes_{A} Y\right)
$$

is an isomorphism.

Given a right continuous functor $G: \bmod -A \rightarrow \bmod -B$ one can recover the bimodule $X$ as follows. Put $X=G(A)$ as right $B$-module. For $a \in A$ let $a$. denote the endomorphism of right $A$-module $A$ given by left multiplication with $a$, then the left $A$-module structure on $X$ is given by $a x=G(a \cdot)(x)$ for $a \in A$ and $x \in X$. Applying this theory to our situation one gets that for two right continuous functors $F$ and $G$ from $\mathcal{A}$ to $\mathcal{B}$ which naturally $\mathfrak{F}$-commute with projective functors one has $\operatorname{Hom}_{F u n c}(F, G) \simeq \operatorname{Hom}_{A-B}(F(\mathcal{P}(M)), G(P(M))$. By Definition 2 the last space is isomorphic to $\operatorname{Hom}_{A-B}(\mathfrak{F}(\mathcal{P})(F(M)), \mathfrak{F}(\mathcal{P})(G(M)))$. Let $t \in A=\operatorname{Hom}_{\mathcal{A}}(P(M), P(M))$ and $\mathfrak{t} \in \operatorname{Hom}_{\text {Func }}(P, P)$ such that $\mathfrak{t}_{M}=t$. The left multiplication of elements from $\mathfrak{F}(\mathcal{P})(F(M))$ by $t$ is given by the endomorphism $\mathfrak{F}(\mathfrak{t})_{F(M)}(x)$, similarly one can describe the left $A$-module structure on $\mathfrak{F}(\mathcal{P})(G(M))$. One can easily see that any morphism of right $B$-modules from $F(M)$ to $G(M)$ induces a morphism of $A-B$ bimodules from $\mathfrak{F}(\mathcal{P})(F(M))$ to $\mathfrak{F}(\mathcal{P})(G(M))$ but, in general, 
not every morphism of these bimodules is induced by a morphism from $F(M)$ to $G(M)$.

Remark 1. Dualizing the notions and arguments of this section one can define categories with full injective functors and functors between two categories which naturally $\mathfrak{F}$-commute with injective functors.

\section{Preliminaries from Representation Theory}

Let $\mathfrak{g} \supset \mathfrak{b} \supset \mathfrak{h}$ be a semisimple finite dimensional Lie algebra over an algebraically closed field $\mathbb{C}$ of characteristic 0 with fixed Borel and Cartan subalgebras. This data provides Cartan (triangular) decomposition $\mathfrak{g}=\mathfrak{n}_{-} \oplus \mathfrak{b}=\mathfrak{n}_{-} \oplus \mathfrak{h} \oplus \mathfrak{n}_{+}$. Let $R=R^{+} \cup R^{-} \subset \mathfrak{h}^{*}$ be the root system of $\mathfrak{g}$ with decomposition into positive and negative roots (corresponding to $\mathfrak{n}_{+}$and $\mathfrak{n}_{-}$) and the basis $\pi \subset R^{+}$. We define $\rho \in \mathfrak{h}^{*}$ by the condition $2 \rho=\sum_{\alpha \in R^{+}} \alpha$. The Weyl group $W$ of $R$ is generated by simple reflections $s_{\alpha}, \alpha \in \pi$. Let $w_{0}$ be the longest element of $W$. Sometimes we will use the dot action of $W$ on $\mathfrak{h}^{*}$ defined by $w \cdot \lambda=w(\lambda+\rho)-\rho$ for $w \in W$ and $\lambda \in \mathfrak{h}^{*}$. For a subset $S \subset \pi$ by $W_{S}$ we denote the subgroup of $W$ generated by $s_{\alpha}, \alpha \in S$. Let $\ell(\cdot)$ denote the standard length function on $W$. Fix a parabolic subalgebra $\mathfrak{p} \subset \mathfrak{g}$ containing $\mathfrak{b}$. The Lie algebra $\mathfrak{p}$ decomposes as a direct sum of the maximal reductive subalgebra $\mathfrak{m} \subset \mathfrak{p}$ and nilpotent radical $\mathfrak{u}$ of $\mathfrak{p}$. By $W_{\mathfrak{p}}$ we denote the subgroup of $W$ generated by reflections $\left\{s_{\alpha} \mid \alpha \in R, \mathfrak{m}_{\alpha} \neq 0\right\}$.

Let $S(\mathfrak{h})$ be the symmetric algebra over $\mathfrak{h}$, and let $Z(\mathfrak{g})$ be the center of the universal enveloping algebra $U(\mathfrak{g})$ of $\mathfrak{g}$. The Harish-Chandra homomorphism $\theta^{\#}: Z(\mathfrak{g}) \rightarrow S(\mathfrak{h})$, normalized by the condition $z-$ $\theta^{\#}(z) \in U\left(\mathfrak{n}_{-}\right)$, induces an isomorphism of $Z(\mathfrak{g})$ onto $S(\mathfrak{h})^{W}$. For $\lambda \in \mathfrak{h}^{*}$ by $|\lambda|$ we will denote the element of $\operatorname{Max}(Z(\mathfrak{g}))$ given by the projection $\theta: \mathfrak{h}^{*} /(W \cdot) \rightarrow \operatorname{Max}(Z(\mathfrak{g}))$. For every $\chi \in \operatorname{Max} Z(\mathfrak{g})$ the canonical projection $Z(\mathfrak{g}) \rightarrow Z(\mathfrak{g}) / \chi=\mathbb{C}$ defines a central character, which will be denoted by the same symbol.

The category $\mathcal{O}$, by definition, is the full subcategory of $\mathfrak{g}$-mod whose objects are all finite generated, $\mathfrak{h}$-diagonalizable, $\mathfrak{n}_{+}$-locally finite $\mathfrak{g}$ modules. The action of $Z(\mathfrak{g})$ provides the central character decomposition $\mathcal{O}=\bigoplus_{\chi \in \operatorname{Max} Z(\mathfrak{g})} \mathcal{O}_{\chi}$ where $\mathcal{O}_{\chi}$ consist of all modules $M \in \mathrm{Ob}(\mathcal{O})$ such that $(z-\chi(z))^{n} M=0$ for $z \in Z(\mathfrak{g})$ and $n \gg 0$. For a module $M \in \operatorname{Ob}(\mathcal{O})$ and $\lambda \in \mathfrak{h}^{*}$ by $M_{\lambda}$ we denote the $\lambda$-weight subspace of $M$. By the definition $M_{\lambda}=\{m \in M \mid(h-\lambda(h)) m=0$ for all $h \in \mathfrak{h}\}$ and $M=\bigoplus_{\lambda \in \mathfrak{h}^{*}} M_{\lambda}$. The most combinatorial difficulty of the category 
$\mathcal{O}$ is encoded in the block $\mathcal{O}_{0}$ (this is the block, containing the trivial $\mathfrak{g}$-module).

We will consider $\mathfrak{g}$ as a $\mathfrak{g}$-module with respect to the adjoint action. For all $\alpha \in R$ there exists a unique element $h_{\alpha} \in\left[\mathfrak{g}_{\alpha}, \mathfrak{g}_{-\alpha}\right] \subset \mathfrak{h}^{*}$ such that $\alpha\left(h_{\alpha}\right)=2$. Let $\mathcal{P}(R)=\left\{\lambda \in \mathfrak{h}^{*} \mid \lambda\left(h_{\alpha}\right) \in \mathbb{Z}\right\}$ be the group of integral weights and let $\mathcal{O}_{\text {int }}=\bigoplus_{\lambda \in P(R)} \mathcal{O}_{|\lambda|}$. A weight $\lambda \in \mathfrak{h}^{*}$ is called dominant if $(\lambda+\rho)\left(h_{\alpha}\right) \notin\{-1,-2, \ldots\}$ for all $\alpha \in R^{+}, \lambda$ is called regular if $(\lambda+\rho)\left(h_{\alpha}\right) \neq 0$ for all $\alpha \in R$. For $\lambda \in \mathfrak{h}^{*}$ let $\mathbb{C}_{\lambda}$ denote the one dimensional $\mathfrak{b}$-module of weight $\lambda$. The Verma module $M(\lambda)=U(\mathfrak{g}) \bigotimes_{U(\mathfrak{b})} \mathbb{C}_{\lambda}$ is an object of category $\mathcal{O}$. It has a unique maximal submodule and the corresponding simple quotient is denoted by $L(\lambda)$. Every simple object of $\mathcal{O}$ is isomorphic to $L(\lambda)$ for some $\lambda \in \mathfrak{h}^{*}$. Category $\mathcal{O}$ has enough projective objects, let $P(\lambda)$ denote the projective cover of $L(\lambda)$. The Verma module $M(\lambda)$ is projective if and only if $\lambda$ is dominant. We denote by $*$ the standard contravariant simple preserving duality functor on $\mathcal{O}$. See [Ja] for details.

Let $\mathcal{F}(\mathfrak{g})$ denote the category of all finite dimensional representations of $\mathfrak{g}$ and let $\mathcal{F}_{0}(\mathfrak{g})$ be the full subcategory of $\mathcal{F}(\mathfrak{g})$ whose objects are all finite dimensional modules $F$ such that the zero weight space of every non-trivial subquotient of $F$ is non-zero.

The category of all finitely generated $\mathfrak{g}$-modules is denoted by $\mathcal{M}$. For an ideal $I$ in $Z(\mathfrak{g})$ by $\mathcal{M}(I)$ (resp. $\mathcal{M}^{\infty}(I)$ ) we will denote the full subcategory of $\mathcal{M}$ whose objects are all modules $M \in \mathcal{M}$ such that $I M=0$ (resp. there exist a positive integer $m$ such that $z^{m} M=0$ for all $z \in I)$. The full subcategory of $\mathcal{M}$ whose objects are all locally $Z(\mathfrak{g})$-finite $\mathfrak{g}$-modules is denoted by $\mathcal{M}_{Z f}$.

For an $U(\mathfrak{g})$ bimodule $X$ let $X^{\text {ad }}$ denote the $\mathfrak{g}$-module which is equal to $X$ as a vector space and the adjoint action of $\mathfrak{g}$ given by $g \cdot x=g x-x g$ for all $g \in \mathfrak{g}$ and $x \in X$. For a $\mathfrak{g}$-module $M$ let $M \otimes X$ denote the $U(\mathfrak{g})-U(\mathfrak{g})$ bimodule which is equal to $M \otimes X$ as a vector space and the right and the left actions of $\mathfrak{g}$ are given by $g(m \otimes x)=(g m) \otimes x+$ $m \otimes(g x),(m \otimes x) g=m \otimes(x g)$ for all $g \in \mathfrak{g}, m \in M$ and $x \in X$. For a right $\mathfrak{g}$-module $M^{\prime}$ the $U(\mathfrak{g})-U(\mathfrak{g})$ bimodule $X \otimes M^{\prime}$ is defined similarly. Let $X^{\text {adf }}$ denote the maximal $U(\mathfrak{g})$-subbimodule of $X$ which decomposes into a direct sum of finite dimensional $\mathfrak{g}$-modules under the adjoint action. The category of Harish-Chandra bimodules $\mathcal{H}$ is the full subcategory of the category of $U(\mathfrak{g})$ bimodules whose objects are all bimodules $X$ which are finite generated as left (or right) $U(\mathfrak{g})$ modules, such that $X=X^{\text {adf }}$. For ideals $I, J$ in $Z(\mathfrak{g})$ by $\mathcal{H}_{I}$ (resp. 
$\mathcal{H}_{I}^{\infty}$ ) we denote the full subcategory of $\mathcal{H}$ whose objects are HarishChandra bimodules $X$ satisfying $X I=0$ (resp. $X I^{m}=0$ for $m \gg 0$ ). Similarly we define ${ }_{I} \mathcal{H},{ }_{I}^{\infty} \mathcal{H},{ }_{I} \mathcal{H}_{J}$ and so on. For two $\mathfrak{g}$-modules $M$ and $N$ the space $\operatorname{Hom}_{\mathbb{C}}(M, N)$ has a structure of $U(\mathfrak{g})-U(\mathfrak{g})$ bimodule, its maximal adjoint finite subbimodule is denoted by $\mathcal{L}(M, N)$. If $\lambda$ is integral regular dominant then the functor $\mathcal{L}\left(M(\lambda),{ }_{-}\right)$induces an equivalence of $\mathcal{O}_{\text {int }}$ and $\mathcal{H}_{|\lambda|}$, the inverse equivalence is given by the functor $-\bigotimes_{U(\mathfrak{g})} M(\lambda)$ (see e.g. [Ja, 6.17]).

Lemma 2 (see [BG, Lemma 2.2]). For an $U(\mathfrak{g})-U(\mathfrak{g})$ bimodule $X$ and a finite dimensional $\mathfrak{g}$-module $E$ there is a natural isomorphism

$$
\operatorname{Hom}_{U(\mathfrak{g})-U(\mathfrak{g})}(E \otimes U(\mathfrak{g}), X) \cong \operatorname{Hom}_{\mathfrak{g}}\left(E, X^{\mathrm{ad}}\right) .
$$

Definition 4 ([BG]). A functor $F: \mathcal{M}_{Z f} \rightarrow \mathcal{M}_{Z f}$ is called projective if it is isomorphic to a direct summand of $\left.\left(E \otimes_{-}\right)\right|_{\mathcal{M}_{Z f}}$ for some finite dimensional $\mathfrak{g}$-module $E$.

The special case of projective functors are translation functors. Let $\lambda, \mu \in \mathfrak{h}^{*}$ be dominant such that $\lambda-\mu$ is integral and $E_{\lambda}^{\mu}$ be a simple finite dimensional $\mathfrak{g}$-module with highest weight in $W(\lambda-\mu)$. The translation functor $T_{\lambda}^{\mu}: \mathcal{M}^{\infty}(|\lambda|) \rightarrow \mathcal{M}^{\infty}(|\mu|)$ is defined by $T_{\lambda}^{\mu}(M)=$ $\operatorname{Pr}_{\mu}(E \otimes M)$ where $\operatorname{Pr}_{\mu}$ denotes the projection on the block with central character $|\mu|$.

Lemma 3. Let $\lambda \in \mathfrak{h}^{*}, \mu \in \lambda+\mathcal{P}(R)$ be dominant, and let $M, N \in$ $\mathcal{M}^{\infty}(|\lambda|)$ with $\operatorname{Ann}_{U(\mathfrak{g})}(M)=\operatorname{Ann}_{U(\mathfrak{g})}(N)$. Then $\operatorname{Ann}_{U(\mathfrak{g})}\left(T_{\lambda}^{\mu}(M)\right)=$ $\operatorname{Ann}_{U(\mathfrak{g})}\left(T_{\lambda}^{\mu}(N)\right)$ in particular $T_{\lambda}^{\mu}(M)=0$ if and only if $T_{\lambda}^{\mu}(N)=0$.

Proof. Denote $E=E_{\lambda}^{\mu}$. Let $\delta: U(\mathfrak{g}) \rightarrow U(\mathfrak{g}) \otimes U(\mathfrak{g})$ be the standard comultiplication map given by $\delta(g)=g \otimes 1+1 \otimes g$ for all $g \in \mathfrak{g}$. One easily gets

$\operatorname{Ann}_{U(\mathfrak{g})}(E \otimes M)=\delta^{-1}\left(\operatorname{Ann}_{U(\mathfrak{g})}(E) \otimes U(\mathfrak{g})+U(\mathfrak{g}) \otimes \operatorname{Ann}_{U(\mathfrak{g})}(M)\right)=: J$.

$\operatorname{Similarly} \operatorname{Ann}(E \otimes N)=J$. From the definition of translation functors it follows that

$$
\operatorname{Ann}_{U(\mathfrak{g})}\left(T_{\lambda}^{\mu}(M)\right)=\bigcap_{n \in \mathbb{N}} J+\theta^{\#}(\mu)^{n}=\operatorname{Ann}_{U(\mathfrak{g})}\left(T_{\lambda}^{\mu}(N)\right) .
$$

In particular $\operatorname{Ann}_{U(\mathfrak{g})}\left(T_{\lambda}^{\mu}(M)\right)=\operatorname{Ann}_{U(\mathfrak{g})}\left(T_{\lambda}^{\mu}(N)\right)$ and the lemma follows.

\section{Some subcategories of $\mathfrak{g}-\mathrm{MOD}$}

To every $\mathfrak{g}$-module $M$ one can associate two full subcategories of $\mathfrak{g}$-mod: $\langle\mathcal{F}(\mathfrak{g}) \otimes M\rangle$ and $\operatorname{coker}(\mathcal{F}(\mathfrak{g}) \otimes M)$. The objects of $\langle\mathcal{F}(\mathfrak{g}) \otimes M\rangle$ 
are all subquotients of the modules $E \otimes M, E \in \mathcal{F}(\mathfrak{g})$. The objects of $\operatorname{coker}(\mathcal{F}(\mathfrak{g}) \otimes M)$ are $\mathfrak{g}$-modules $N$ that fit into an exact sequence of the form $E \otimes M \rightarrow F \otimes M \rightarrow N \rightarrow 0$ for some $E, F \in \mathcal{F}(\mathfrak{g})$. Similarly one can define the categories $\left\langle\mathcal{F}_{0}(\mathfrak{g}) \otimes M\right\rangle$ and $\operatorname{coker}\left(\mathcal{F}_{0}(\mathfrak{g}) \otimes M\right)$.

For technical reasons we assume that $M$ has a (generalized) central character, is finitely generated and projective in $\langle\mathcal{F}(\mathfrak{g}) \otimes M\rangle$. In this case the category $\operatorname{coker}(\mathcal{F}(\mathfrak{g}) \otimes M)$ decomposes into blocks with respect to the action of the center, has enough projective objects (only finite many up to isomorphism in each block). It is abelian, because it is equivalent (blockwise) to a category of modules over associative algebra. The $\mathfrak{g}$-module structure of objects of $\langle\mathcal{F}(\mathfrak{g}) \otimes M\rangle$ is completely encoded in this category, however the category $\operatorname{coker}(\mathcal{F}(\mathfrak{g}) \otimes M)$ is in many cases easier to deal with. For example, for regular dominant $\lambda \in \mathfrak{h}^{*}$ the category $\langle\mathcal{F}(\mathfrak{g}) \otimes M(\lambda)\rangle$ coincides with $\operatorname{coker}(\mathcal{F}(\mathfrak{g}) \otimes M(\lambda))$ (if $\lambda$ is integral they both are equal to $\mathcal{O}_{\text {int }}$ ). If $\lambda$ is not regular, then $\operatorname{coker}(\mathcal{F}(\mathfrak{g}) \otimes M(\lambda))$ is a proper subcategory of $\langle\mathcal{F}(\mathfrak{g}) \otimes M(\lambda)\rangle$, and simple objects of $\operatorname{coker}(\mathcal{F}(\mathfrak{g}) \otimes M(\lambda))$ are not necessary simple $\mathfrak{g}$-modules.

For $\chi \in \operatorname{Max}(Z(\mathfrak{g}))$ let $P_{\chi}^{M}$ be a projective generator of $\operatorname{coker}(\mathcal{F}(\mathfrak{g}) \otimes$ $M)_{\chi}$ which is also projective in $\langle\mathcal{F}(\mathfrak{g}) \otimes M\rangle$ (it exists because of our assumptions on $M)$. By abstract arguments we get an equivalence of categories $\operatorname{coker}(\mathcal{F}(\mathfrak{g}) \otimes M)_{\chi} \cong \bmod -\operatorname{End}_{\mathfrak{g}}\left(P_{\chi}^{M}\right)$ given by functors $\operatorname{Hom}_{\mathfrak{g}}\left(P_{\chi}^{M},{ }_{-}\right)$and $P_{\chi}^{M} \bigotimes_{\operatorname{End}_{\mathfrak{g}}\left(P_{\chi}^{M}\right)-}$ (see $[\mathrm{Au}, 5.1]$ for details $)$. The functor $P_{\chi}^{M} \bigotimes_{\operatorname{End}_{\mathfrak{g}}\left(P_{\chi}^{M}\right)} \operatorname{Hom}_{\mathfrak{g}}\left(P_{\chi}^{M},{ }_{-}\right):\langle\mathcal{F}(\mathfrak{g}) \otimes M\rangle \rightarrow \operatorname{coker}(\mathcal{F}(\mathfrak{g}) \otimes M)$ is plainly right adjoint to $\mathfrak{i}$. This functor will be denoted by $\tilde{\mathcal{C}}_{M}$, and will be called coapproximation functor. Sometimes, if there is no risk of confusion, we omit the subscript $M$.

Lemma 4. The functor $\tilde{\mathcal{C}}$ is exact.

Proof. the functor $\operatorname{Hom}_{\mathfrak{g}}\left(P_{\chi}^{M},{ }_{-}\right)$is exact because $P_{\chi}^{M}$ is projective in $\langle\mathcal{F}(\mathfrak{g}) \otimes M\rangle$ and the functor $P_{\chi}^{M} \bigotimes_{\operatorname{End}_{\mathfrak{g}}\left(P_{\chi}^{M}\right)}$ is an equivalence of abelian categories.

The trace of a module $N_{1}$ in a module $N_{2}$ is by definition the sum of images of all morphisms $N_{1} \rightarrow N_{2}$. For $K \in \mathrm{Ob}\langle\mathcal{F}(\mathfrak{g}) \otimes M\rangle$ let $\hat{K}$ be the sum of traces of all modules $E \otimes M, E \in \mathcal{F}(\mathfrak{g})$, and let $\phi_{K}: \mathcal{P}_{K} \rightarrow \hat{K}$ be its projective cover by finite direct sum of modules of type $E \otimes M, E \in \mathcal{F}(\mathfrak{g})$. Then the value of $\tilde{\mathcal{C}}$ on $N \in \mathrm{Ob}\langle\mathcal{F}(\mathfrak{g}) \otimes M\rangle$ is isomorphic to $\tilde{\mathcal{C}}(N) \cong \mathcal{P}_{N} / \phi_{\operatorname{ker} \phi_{N}}\left(\mathcal{P}_{\operatorname{ker} \phi_{N}}\right)$.

Obviously the canonical morphism $\operatorname{Id}_{\operatorname{coker}(\mathcal{F}(\mathfrak{g}) \otimes M)} \rightarrow \tilde{\mathcal{C}} \circ \mathfrak{i}$ is an isomorphism, thus, by abstract nonsense (see [Ga] or [BeGi, Lemma 2.4]), the functor $\tilde{\mathcal{C}}$ induces an equivalence of categories $\langle\mathcal{F}(\mathfrak{g}) \otimes M\rangle / \operatorname{ker} \tilde{\mathcal{C}} \rightarrow$ 
$\operatorname{coker}(\mathcal{F}(\mathfrak{g}) \otimes M)$. In the sequel we will need the following technical lemma.

Lemma 5. Let $N \in \mathrm{Ob}\langle\mathcal{F}(\mathfrak{g}) \otimes M\rangle$ be such that the canonical morphism can : io $\tilde{\mathcal{C}}(N) \rightarrow N$ is surjective, then for any $K \in \mathrm{Ob}\langle\mathcal{F}(\mathfrak{g}) \otimes M\rangle$ the canonical map $\operatorname{Hom}_{\mathfrak{g}}(N, K) \rightarrow \operatorname{Hom}_{\mathfrak{g}}(\tilde{\mathcal{C}}(N), \tilde{\mathcal{C}}(K))$ is injective.

Proof. Let $0 \neq \phi \in \operatorname{Hom}_{\mathfrak{g}}(N, K)$. By assumption $\phi \circ$ can is non-zero. It is easy to see that under the canonical isomorphism $\operatorname{Hom}_{\mathfrak{g}}(\mathfrak{i} \tilde{\mathcal{C}}(N), K) \cong$ $\operatorname{Hom}_{\mathfrak{g}}(\tilde{\mathcal{C}}(N), \tilde{\mathcal{C}}(K))$ the map $\phi \circ$ can corresponds to $\tilde{\mathcal{C}}(\phi) \circ i d=\tilde{\mathcal{C}}(\phi)$, which proves the lemma.

Let us briefly describe the dual construction. If $\tilde{M}$ is a $\mathfrak{g}$-module one can define, $\operatorname{ker}(\mathcal{F}(\mathfrak{g}) \otimes \tilde{M})$ to be the full subcategory of $\mathfrak{g}$-modules whose objects are modules $\tilde{N}$ that fit into an exact sequence of the form $0 \rightarrow \tilde{N} \rightarrow E \otimes \tilde{M} \rightarrow F \otimes \tilde{M}$ for some finite dimensional $\mathfrak{g}$-modules $E$ and $F$. If $\tilde{M}$ is injective in $\langle\mathcal{F}(\mathfrak{g}) \otimes \tilde{M}\rangle$ then $\operatorname{ker}(\mathcal{F}(\mathfrak{g}) \otimes \tilde{M})$ is abelian and one can construct the left adjoint functor $\mathcal{C}_{\tilde{M}}:\langle\mathcal{F}(\mathfrak{g}) \otimes \tilde{M}\rangle \rightarrow$ $\operatorname{ker}(\mathcal{F}(\mathfrak{g}) \otimes \tilde{M})$ to the inclusion functor. The functor $\mathcal{C}$ is exact and induces an equivalence $\langle\mathcal{F}(\mathfrak{g}) \otimes \tilde{M}\rangle / \operatorname{ker} \mathcal{C} \cong \operatorname{ker}(\mathcal{F}(\mathfrak{g}) \otimes \tilde{M})$. It is called the approximation functor.

For $\chi \in \operatorname{Max}(Z(\mathfrak{g}))$ define $\langle\mathcal{F}(\mathfrak{g}) \otimes M\rangle_{\chi}=\langle\mathcal{F}(\mathfrak{g}) \otimes M\rangle \cap \mathcal{M}^{\infty}(\chi)$ and $\operatorname{coker}(\mathcal{F}(\mathfrak{g}) \otimes M)_{\chi}=\operatorname{coker}(\mathcal{F}(\mathfrak{g}) \otimes M) \cap \mathcal{M}^{\infty}(\chi)$ respectively.

Proposition 3. ( $M$ is projective in $\langle\mathcal{F}(\mathfrak{g}) \otimes M\rangle$ and has a generalized central character.) Suppose that for any $F \in \mathrm{Ob} \mathcal{F}(\mathfrak{g})$ the canonical map

$$
\operatorname{Hom}_{g}\left(F, U(\mathfrak{g})^{\mathrm{ad}}\right) \rightarrow \operatorname{Hom}_{\mathfrak{g}}\left(F,\left(\operatorname{End}_{\mathbb{C}}(M)^{\mathrm{adf}}\right)^{\mathrm{ad}}\right)
$$

given by multiplication is surjective. Then

(1) The category coker $(\mathcal{F}(\mathfrak{g}) \otimes M)$ together with the dominant object $M$ and all direct summands of functors

$$
\left\{F \otimes P r_{\chi}(-) \mid F \in \mathrm{Ob}(\mathcal{F}(\mathfrak{g}))\right\} \cup\{\mathbf{I d}\}
$$

is a category with full projective functors.

(2) An object $N$ of $\operatorname{coker}(\mathcal{F}(\mathfrak{g}) \otimes M)$ is quasidominant if and only if $\operatorname{Ann}_{U(\mathfrak{g})} N \supset \operatorname{Ann}_{U(\mathfrak{g})} M$.

(3) Let $M^{\prime}$ be another $\mathfrak{g}$-module satisfying the assumptions of this proposition. Suppose $\mathrm{Ann}_{U(\mathfrak{g})} M=\mathrm{Ann}_{U(\mathfrak{g})} M^{\prime}$, then the categories $\operatorname{coker}(\mathcal{F}(\mathfrak{g}) \otimes M)$ and $\operatorname{coker}\left(\mathcal{F}(\mathfrak{g}) \otimes M^{\prime}\right)$ are equivalent.

Proof. It is enough to check the requirements of Definition 1 only for functors $\left\{F \otimes \operatorname{Pr}_{\chi}(-) \mid F \in \mathrm{Ob}(\mathcal{F}(\mathfrak{g}))\right\} \cup\{\mathbf{I d}\}$. The Conditions (PF1), $(\mathrm{PF} 2)$ and (PF3) are obvious. 
Let $I=\operatorname{Ann}_{U(\mathfrak{g})} M, \chi=Z(\mathfrak{g}) \cap I$ and $U^{n}=U(\mathfrak{g}) / U(\mathfrak{g}) \chi^{n}$. Let $E_{1}$ and $E_{2}$ be two finite dimensional $\mathfrak{g}$-modules. For arbitrary positive integer $n$ we get by Theorem 6 and Lemma 2

$$
\begin{aligned}
& \operatorname{Hom}_{F u n c}\left(\left.\left(E_{1} \otimes{ }_{-}\right)\right|_{\mathcal{M}\left(\chi^{n}\right)},\left.\left(E_{2} \otimes-\right)\right|_{\mathcal{M}\left(\chi^{n}\right)}\right) \cong \\
& \operatorname{Hom}_{U(\mathfrak{g})-U(\mathfrak{g})}\left(E_{1} \otimes U^{n}, E_{2} \otimes U^{n}\right) \cong \operatorname{Hom}_{U(\mathfrak{g})-U(\mathfrak{g})}\left(E_{1} \otimes U(\mathfrak{g}), E_{2} \otimes U^{n}\right) \cong \\
& \operatorname{Hom}_{\mathfrak{g}}\left(E_{1} \otimes E_{2}^{*},\left(U^{n}\right)^{\mathrm{ad}}\right) .
\end{aligned}
$$

The functor $\operatorname{Hom}_{\mathfrak{g}}\left(E_{1} \otimes E_{2}^{*},{ }_{-}\right)$is exact on the category of locally finite $\mathfrak{g}$-modules, thus the natural projection $U^{n} \rightarrow U / I$ together with the isomorphism above provides a surjective map

$$
\operatorname{Hom}_{\text {Func }}\left(\left.\left(E_{1} \otimes_{-}\right)\right|_{\mathcal{M}^{\infty}(\chi)},\left.\left(E_{2} \otimes_{-}\right)\right|_{\mathcal{M}^{\infty}(\chi)}\right) \rightarrow \operatorname{Hom}_{\mathfrak{g}}\left(E_{1} \otimes E_{2}^{*}, U / I\right) .
$$

By the assumption the last space is isomorphic to

$$
\operatorname{Hom}_{\mathfrak{g}}\left(E_{1} \otimes E_{2}^{*}, \operatorname{End}_{\mathbb{C}}(M)^{\mathrm{ad}}\right) \cong \operatorname{Hom}_{\mathfrak{g}}\left(E_{1} \otimes M, E_{2} \otimes M\right)
$$

(the isomorphism is by [Ja, 6.8(3)]). This proves the first statement of the proposition.

Let now $\mathfrak{t} \in \operatorname{Hom}_{U(\mathfrak{g})-U(\mathfrak{g})}\left(E_{1} \otimes U^{n}, E_{2} \otimes U^{n}\right)$. It defines a morphism of $\mathfrak{g}$-modules $\mathfrak{t}_{M}: E_{1} \otimes M \rightarrow E_{2} \otimes M$. Let $\left(v_{i}\right)_{i \in \mathcal{I}}$ and $\left(v_{j}^{\prime}\right)_{j \in \mathcal{J}}$ be bases of $E_{1}$ and $E_{2}$ respectively, and let $\left(u_{i j}\right)_{i \in \mathcal{I}, j \in \mathcal{J}} \in U^{n}$ be given by the condition $\mathfrak{t}\left(v_{i} \otimes 1\right)=\sum_{j \in \mathcal{J}} v_{j} \otimes u_{i j}$ for all $i \in \mathcal{I}$. In particular for all $m \in M$ and $i \in \mathcal{I}$ one has $\mathfrak{t}_{M}\left(v_{i} \otimes m\right)=\sum_{j \in \mathcal{J}} v_{j} \otimes u_{i j} m$.

Since $\left(v_{j}^{\prime}\right)_{j \in \mathcal{J}}$ are linearly independent we get that if $\mathfrak{t}_{M}=0$ then all $u_{i j} \in \operatorname{Ann}_{U(\mathfrak{g})} M$. This implies that if $\operatorname{Ann}_{U(\mathfrak{g})} M \subset \operatorname{Ann}_{U(\mathfrak{g})} N$ then $\mathfrak{t}_{N}=0$ and $N$ is quasidominant.

Suppose now $I=\operatorname{Ann}_{U(\mathfrak{g})} M \not \subset \operatorname{Ann}_{U(\mathfrak{g})} N=: J$. Then there exist a finite dimensional module $E$ and a non-zero $U(\mathfrak{g})$-bimodule morphism $E \otimes U(\mathfrak{g}) \rightarrow I / I \cap J$. It can be lifted to an $U(\mathfrak{g})$-bimodule morphism $\mathfrak{f}: E \otimes U(\mathfrak{g}) \rightarrow I$ such that $\mathfrak{f}(E \otimes U(\mathfrak{g})) \not \subset J$ (this morphism obviously defines a morphism of projective functors). Let $v \in E$ be such that $\mathfrak{f}(v \otimes 1) \notin J$. Again the morphism $\mathfrak{f}$ defines morphisms of modules $\mathfrak{f}_{M}: E \otimes M \rightarrow M$ and $\mathfrak{f}_{N}: E \otimes N \rightarrow N$. And for every $m \in M, n \in N$ one has $\mathfrak{f}_{M}(v \otimes m)=\mathfrak{f}(v \otimes 1) m$ and $\mathfrak{f}_{N}(v \otimes n)=\mathfrak{f}(v \otimes 1) n$. By the construction $\mathfrak{f}(v \otimes 1) \in I \backslash J$ thus $\mathfrak{f}_{M}=0$ but $\mathfrak{f}_{N} \neq 0$. This implies that $N$ is not quasidominant and proves the second statement of the proposition. The third statement follows now from Corollary 1.

Remark 2. Proposition 3 is true if one replaces $\mathcal{F}(\mathfrak{g})$ by $\mathcal{F}_{0}(\mathfrak{g})$.

Restricting Proposition 3 to modules having a central character we get the following corollary. 
Corollary 2. Let $M$ and $M^{\prime}$ be two $\mathfrak{g}$-modules as in Proposition 3 such that $\operatorname{Ann}_{U(\mathfrak{g})} M=\operatorname{Ann}_{U(\mathfrak{g})} M^{\prime} \supset \chi U(\mathfrak{g})$ for some $\chi \in \operatorname{Max}(Z(\mathfrak{g}))$.

(1) The category coker $(\mathcal{F}(\mathfrak{g}) \otimes M)_{\chi}$ together with the dominant object $M$ and projective functors (from Definition 4) is a category with full projective functors.

(2) An object $N$ of $\operatorname{coker}(\mathcal{F}(\mathfrak{g}) \otimes M)_{\chi}$ is quasi-dominant if and only if $\operatorname{Ann}_{U(\mathfrak{g})} N \supset \operatorname{Ann}_{U(\mathfrak{g})} M$.

(3) The categories $\operatorname{coker}(\mathcal{F}(\mathfrak{g}) \otimes M)_{\chi}$ and $\operatorname{coker}\left(\mathcal{F}(\mathfrak{g}) \otimes M^{\prime}\right)_{\chi}$ are equivalent.

\section{Category $\mathcal{O}$}

In this section we will study the right exact additive endofunctors on (blocks of) category $\mathcal{O}$ which naturally commute with projective functors with respect to the identity functor on $\operatorname{Hom}(\mathcal{O}, \mathcal{O})$. For simplicity we will work only with integral blocks, the results however can be transferred to non-integral case by introducing enough indexes.

5.1. Projective functors on $\mathcal{O}$. The category $\mathcal{O}_{\text {int }}$ is equal to the category $\operatorname{coker}(\mathcal{F}(\mathfrak{g}) \otimes M(\lambda))$ for a dominant integral regular weight $\lambda \in \mathfrak{h}^{*}$. The next proposition is now a special case of Proposition 3 and Corollary 2.

Proposition 4. Let $\lambda \in \mathfrak{h}^{*}$ be a dominant integral regular weight. The category $\mathcal{O}_{\text {int }}$ together with the dominant object $M(\lambda)$ and all direct summands of the functors $\left\{F \otimes \operatorname{Pr}_{\lambda}(-) \mid F \in \operatorname{Ob}(\mathcal{F}(\mathfrak{g}))\right\} \cup\{\mathbf{I d}\}$ is a category with full projective functors. The category $\mathcal{O}_{|\lambda|}$ together with the dominant object $M(\lambda)$ and set of projective endofunctors of $\mathcal{O}_{|\lambda|}$ from Definition 4 is a category with full projective functors. A module $N$ is a quasi-dominant object of $\mathcal{O}_{\text {int }}\left(\right.$ or $\left.\mathcal{O}_{|\lambda|}\right)$ if and only if $\operatorname{Ann}_{U(\mathfrak{g})} N \supset \operatorname{Ann}_{U(\mathfrak{g})} M(\lambda)$.

Let $\underline{\mathcal{O}}_{\text {int }}$ denote the category with full projective functors

$$
\left(\mathcal{O}_{\text {int }}, M(\lambda),\left\{E \otimes \operatorname{Pr}_{\lambda}(-) \mid E \in \mathrm{Ob}(\mathcal{F})\right\}\right) .
$$

Twisting the above construction by the duality $*$ on $\mathcal{O}$ one also gets that the triple

$$
\underline{\mathcal{O}}_{i n t}^{*}=\left(\mathcal{O}_{i n t}, M(\lambda)^{*},\left\{E \otimes \operatorname{Pr}_{\lambda}(-) \mid E \in \mathrm{Ob}(\mathcal{F})\right\}\right)
$$

is a category with full injective functors. Let us give some well known examples of functors on (blocks of) $\mathcal{O}$ which naturally commute with projective functors. 
5.2. Zuckerman functors. Recall parabolic subalgebra $\mathfrak{p}$ from the Section 3. The Zuckerman functor $\Gamma_{\mathfrak{p}}$ is, by definition, the functor on $\mathcal{O}$ that to a module $M \in \mathrm{Ob}(\mathcal{O})$ associates its maximal locally $U(\mathfrak{p})$-finite submodule. A complete exposition of Zuckerman functors can be found, for instance, in $[\mathrm{KV}]$. Zuckerman functors are left exact and naturally commute with projective (and injective) functors. (This follows from the fact that there exist a natural transformation from the Zuckerman functors to the identity functor which is injective on the objects, see also $[\mathrm{KV}]$.) So they would fit into the picture dual to one described in Section 2. Another possibility to deal with Zuckerman functors is to consider their twists by duality $* \circ \Gamma_{\mathfrak{p}} \circ *$ which are right exact. By definition, the functor $* \circ \Gamma_{\mathfrak{p}} \circ *$ associates to a module $M \in$ $\mathrm{Ob}(\mathcal{O})$ its maximal locally $U(\mathfrak{p})$-finite quotient. Thus it corresponds to the maximal $U(\mathfrak{p})$-finite quotient of $M(\lambda)$ under the bijection given by Theorem 5 . For a subset $S \subset \pi$ by $\Gamma_{S}$ we denote the Zuckerman functor associated to the parabolic Lie subalgebra of $\mathfrak{g}$ generated by $\mathfrak{h}$, $\mathfrak{n}_{-}$and $\left\{\mathfrak{g}_{\alpha} \mid \alpha \in S\right\}$.

5.3. Joseph's version of completion functor. Let $\alpha \in R$ be a simple root, $\lambda$ regular dominant integral and $N \in \mathrm{Ob}(\mathcal{O})$. The Joseph's version of Enright's completion functor (see [J82]) is defined by

$$
\mathcal{C}_{\alpha}^{J}(N)=\mathcal{L}\left(M\left(s_{\alpha} \lambda\right), N\right) \bigotimes_{U(\mathfrak{g})} M(\lambda)
$$

From the natural isomorphism $\mathcal{L}\left(M(\lambda), \mathcal{C}_{\alpha}^{J}(N)\right) \cong \mathcal{L}\left(M\left(s_{\alpha} \lambda\right), N\right)$ one easily gets

$$
\mathcal{L}\left(M(\lambda), E \otimes \mathcal{C}_{\alpha}^{J}(N)\right) \cong E \otimes \mathcal{L}\left(M(\lambda), \mathcal{C}_{\alpha}^{J}(N)\right) \cong E \otimes \mathcal{L}\left(M\left(s_{\alpha} \lambda\right), N\right)
$$

for $E \in \mathcal{F}$. Together with an obvious observation that $\mathcal{C}_{\alpha}^{J}$ preserves $\mathcal{O}_{|\lambda|}$, this implies that $\mathcal{C}_{\alpha}^{J}$ naturally commutes with projective functors (both on $\mathcal{O}$ and on $\mathcal{O}_{|\lambda|}$ ). The functors $\mathcal{C}_{\alpha}^{J}$ satisfy braid relations for different $\alpha$ (see [J83, Corollary 2.9]). For $w \in W$ define $\mathcal{C}_{w}^{J}=\mathcal{C}_{\alpha_{1}}^{J} \circ \cdots \circ$ $\mathcal{C}_{\alpha_{n}}^{J}$ for a reduced decomposition $w=s_{\alpha_{1}} \ldots s_{\alpha_{n}} \in W$. These functors are left exact and thus, by the dual of Theorem 5 , their restrictions to $\mathcal{O}_{|\lambda|}$ are determined up to an isomorphism by their values on $M(\lambda)^{*}$. On the other hand one can consider the right exact twists $* \circ \mathcal{C}_{w}^{J} \circ *$ by duality which are determined up to isomorphisms by their values on $M(\lambda)$.

5.4. Coapproximation and functor $\mathbb{V}$. The category $\mathcal{O}_{\text {int }}$ equals to $\langle\mathcal{F}(\mathfrak{g}) \otimes M(-\rho)\rangle$. The inclusion functors $\mathfrak{i}: \operatorname{coker}(\mathcal{F}(\mathfrak{g}) \otimes M(-\rho)) \rightarrow$ 
$\langle\mathcal{F}(\mathfrak{g}) \otimes M(-\rho)\rangle$ and $\tilde{\mathfrak{i}}: \operatorname{ker}(\mathcal{F}(\mathfrak{g}) \otimes M(-\rho)) \rightarrow\langle\mathcal{F}(\mathfrak{g}) \otimes M(-\rho)\rangle$ naturally commute with projective functors, thus the coapproximation $\tilde{\mathcal{C}}_{M(\rho)}$ and approximation $\mathcal{C}_{M(\rho)}$ functors also naturally commute with projective functors because they are right resp. left adjoint to inclusions. The antidominant projective modules $P\left(w_{0} \cdot \lambda\right)$ ( $\lambda$ integral dominant) are objects of $\operatorname{coker}(\mathcal{F}(\mathfrak{g}) \otimes M(-\rho))$ and $\operatorname{ker}(\mathcal{F}(\mathfrak{g}) \otimes M(-\rho))$, thus, by adjunction, Soergel's combinatoric functor $\mathbb{V}:=\operatorname{Hom}_{\mathfrak{g}}\left(P\left(w_{0} \cdot \lambda\right),{ }_{-}\right)$ (see $[\mathrm{S} 90])$ is isomorphic to the functor $\operatorname{Hom}_{\mathfrak{g}}\left(P\left(w_{0} \cdot \lambda\right), \tilde{\mathcal{C}}_{M(-\rho)}(-)\right)$. So the functor $\mathbb{V}$ is the composition of the coapproximation functor $\tilde{\mathcal{C}}_{M(-\rho)}: \mathcal{O}_{|\lambda|} \rightarrow \operatorname{coker}(\mathcal{F}(\mathfrak{g}) \otimes M(-\rho))_{|\lambda|}$ and the functor

$\operatorname{Hom}_{\mathfrak{g}}\left(P\left(w_{0} \cdot \lambda\right),{ }_{-}\right): \operatorname{coker}(\mathcal{F}(\mathfrak{g}) \otimes M(-\rho))_{|\lambda|} \rightarrow \bmod -\operatorname{End}\left(P\left(w_{0} \cdot \lambda\right)\right)$.

The category $\operatorname{coker}(\mathcal{F}(\mathfrak{g}) \otimes M(-\rho))_{|\lambda|}$ is the full subcategory consisting of modules presentable by $P\left(w_{0} \cdot \lambda\right)$, thus the last functor is an equivalence of categories. This means that essential properties of $\mathbb{V}$ are encoded in the coapproximation functor.

For instance, the injective module $M(\lambda)^{*}$ is $P\left(w_{0} \cdot \lambda\right)$-presentable (by dual version of [S90, Lemma 7]), thus every injective module in $\mathcal{O}_{|\lambda|}$ is $P\left(w_{0} \cdot \lambda\right)$-presentable. The coapproximation functor is obviously full and faithful on the $P\left(w_{0} \cdot \lambda\right)$-presentable modules. This provides a short and elegant proof of Struktursatz [S90] claiming that $\mathbb{V}$ is fully faithful on injective modules (using selfduality of $P\left(w_{0} \cdot \lambda\right)$ this also implies that $\mathbb{V}$ is fully faithful on projectives).

\subsection{Arkhipov's functor and Deodhar's version of completion}

functor. For $\alpha \in \pi$ let $x_{ \pm \alpha}$ be non-zero elements in $\mathfrak{g}_{ \pm \alpha}$ such that $\left[h_{\alpha}, x_{ \pm \alpha}\right]= \pm 2 x_{ \pm \alpha}$ and $\left[x_{\alpha}, x_{-\alpha}\right]=h_{\alpha}$. By $U_{(\alpha)}$ we denote the localization of $U(\mathfrak{g})$ by the multiplicative Ore subset $\left\{x_{-\alpha}^{k} \mid k \in \mathbb{Z}, k \geq 0\right\}$. Deodhar's version of Enright's completion functor is a functor $\mathcal{C}_{\alpha}^{D}$ which to a module $N \in \mathrm{Ob}(\mathcal{O})$ associates the locally $x_{\alpha}$-finite part of the $\mathfrak{g}$ module $U_{\alpha} \bigotimes_{U(\mathfrak{g})} N$. The functors $\mathcal{C}_{\alpha}^{D}$ do not satisfy the braid relations on $\mathcal{O}_{|\lambda|}$.

Arkhipov's functors $\mathcal{A}_{\alpha}$ (see $[\mathrm{Ar}]$ ) are defined by

$$
\mathcal{A}_{\alpha}(M)=\tau_{\alpha}\left(\left(U_{(\alpha)} / U(\mathfrak{g})\right) \bigotimes_{U(\mathfrak{g})} M\right)
$$

where $\tau_{\alpha}$ denotes the twist by an automorphism of $\mathfrak{g}$ corresponding to $s_{\alpha}$. Arkhipov's functors satisfy braid relations (several different proofs of this statement are known, see for example [Ar, An, KM1]). So the definition $\mathcal{A}_{w}=\mathcal{A}_{\alpha_{1}} \circ \cdots \circ \mathcal{A}_{\alpha_{n}}$ for a reduced decomposition $w=$ $s_{\alpha_{1}} \ldots s_{\alpha_{n}} \in W$ of $w$ makes sense. Another possibility to define $\mathcal{A}_{w}$ 
for $w \in W$ (which is actually the original one) is as follows. Let $\mathfrak{n}_{w}=\mathfrak{n}_{-} \cap w^{-1}\left(\mathfrak{n}_{+}\right)$. The $\mathbb{Z}$-grading on $\mathfrak{g}$ defined by $\mathfrak{g}_{1}=\bigoplus_{\beta \in \pi} \mathfrak{g}_{\beta}$ induces uniquely a grading on $U\left(n_{w}\right)$. Define $U\left(\mathfrak{n}_{w}\right)^{\circledast}$ to be the graded dual of $U\left(\mathfrak{n}_{w}\right)$ i.e. $\left(U\left(\mathfrak{n}_{w}\right)^{\circledast}\right)_{i}=\left(U\left(\mathfrak{n}_{w}\right)_{-i}\right)^{*}$. One can define a $U(\mathfrak{g})$ bimodule structure on $S_{w}:=U(\mathfrak{g}) \bigotimes_{U\left(\mathfrak{n}_{w}\right)} U\left(\mathfrak{n}_{w}\right)^{\circledast}$ (see e.g. [An, S97]). The functors $\mathcal{A}_{w}$ can now be defined by $\mathcal{A}_{w}(M)=\tau_{w}\left(S_{w} \bigotimes_{U(\mathfrak{g})} M\right)$ where $\tau_{w}$ denotes the twist by an automorphism of $\mathfrak{g}$ corresponding to $w$.

The following statement was proved by Andersen and Stroppel.

Proposition 5 ([AnS, Theorem 3.2]). For any finite dimensional $\mathfrak{g}$ module $E$, the functors $U_{\alpha} \otimes_{U(\mathfrak{g})}\left(E \otimes_{-}\right)$and $E \otimes\left(U_{\alpha} \otimes_{U_{(\mathfrak{g})}}\right)$ are isomorphic.

Let $\mathfrak{t}^{E}: U_{\alpha} \bigotimes_{U(\mathfrak{g})}\left(E \otimes_{-}\right) \rightarrow E \otimes\left(U_{\alpha} \bigotimes_{U(\mathfrak{g})-}\right)$ be the isomorphism given by the above proposition, $F$ be another finite dimensional $\mathfrak{g}$ module, $N \in \mathrm{Ob}(\mathcal{O})$ and $\mathfrak{f} \in \operatorname{Hom}_{\text {Func }}\left(E \otimes_{-}, F \otimes_{-}\right)$a natural transformation. To prove that the functor $G:=U_{\alpha} \bigotimes_{U(\mathfrak{g})-}: \mathcal{O}_{|\lambda|} \rightarrow \mathfrak{g}$-mod naturally commutes with $E \otimes \otimes_{-}$we have to show that for all nonnegative integer $n, m \in N$ and $e \in E$ holds

$$
\mathfrak{f}_{G(N)}\left(\mathfrak{t}_{N}^{E}\left(x_{-\alpha}^{-n} \otimes e \otimes m\right)\right)=\mathfrak{t}_{N}^{F}\left(G\left(\mathfrak{f}_{N}\right)\left(x_{-\alpha}^{-n} \otimes e \otimes m\right)\right) .
$$

Multiplying by $x_{-\alpha}^{n}$ one gets that this is equivalent to

$$
\mathfrak{f}_{G(N)}\left(\mathfrak{t}_{N}^{E}(1 \otimes e \otimes m)\right)=\mathfrak{t}_{N}^{F}\left(G\left(\mathfrak{f}_{N}\right)(1 \otimes e \otimes m)\right)
$$

(because the module $U_{\alpha} \bigotimes_{U(\mathfrak{g})}(E \otimes N)$ is $x_{-\alpha}$-torsion free). The module $1 \otimes E \otimes N$ is naturally a submodule of $U_{\alpha} \otimes_{U(\mathfrak{g})}(E \otimes N)$. The map $\mathfrak{t}_{N}^{E}$ is given by

$$
x_{-\alpha}^{-n} \otimes(e \otimes m) \mapsto \sum_{k \geq 0}(-1)^{k}\left(\begin{array}{c}
n+k-1 \\
k
\end{array}\right) x_{-\alpha}^{k} e \otimes x_{-\alpha}^{-n-k} \otimes m
$$

(see $[\mathrm{AnS}])$. In particular $\left.\mathfrak{t}_{N}^{E}\right|_{E \otimes N}=\left.\mathfrak{t}_{N}^{F}\right|_{E \otimes N}=\mathbf{I d}$ and $\left.\mathfrak{f}_{G(N)}\right|_{E \otimes N}=$ $\left.G\left(\mathfrak{f}_{N}\right)\right|_{E \otimes N}$. This implies that $U_{\alpha} \bigotimes_{U(\mathfrak{g})}$ - naturally commutes with tensor products with finite dimensional modules.

Since taking locally $x_{\alpha}$-finite part of dense $x_{-\alpha}$-torsion free $\mathfrak{g}$-modules obviously naturally commutes with tensoring with finite dimensional modules, we have shown that Deodhar's version of Enright's completion functors naturally commute with projective functors. These functors are left exact, so they are determined up to an isomorphism by $\mathcal{C}_{\alpha}^{D}\left(M\left(\lambda^{*}\right)\right)$ the last module is isomorphic to the extension of the module $M(\lambda) / M\left(s_{\alpha} \lambda\right)$ by $M\left(s_{\alpha} \lambda\right)$. 
Lemma 6. The functor $\tau_{\alpha}: \mathcal{O}_{|\lambda|} \rightarrow \mathcal{M}^{\infty}(|\lambda|)$ naturally commutes with projective functors on $\mathcal{O}_{|\lambda|}$.

Proof. For a right, (resp. left) $\mathfrak{g}$-module $M$, by $M^{\tau}$ (resp. $\left.{ }^{\tau} M\right)$ we will denote a $\mathfrak{g}$-module which is equal to $M$ as vector space, and the right (resp. left) action of $\mathfrak{g}$ is twisted by an endomorphism of $\mathfrak{g}$ corresponding to $s_{\alpha}$. The functor $\tau_{\alpha}$ obviously commutes with projective functors. It is enough to prove that $\tau_{\alpha}$ naturally commutes with $E \otimes_{-}$ for finite dimensional $\mathfrak{g}$-module $E$. Let $\lambda$ be an integral regular dominant weight. Every morphism of functors $E \otimes_{-} \rightarrow F \otimes_{-}(E$ and $F$ are finite dimensional $\mathfrak{g}$-modules) on $\mathcal{O}_{|\lambda|}$ comes from a morphism of bimodules $f: E \otimes\left(U(\mathfrak{g}) /|\lambda|^{n} U(\mathfrak{g})\right) \rightarrow F \otimes\left(U(\mathfrak{g}) /|\lambda|^{n} U(\mathfrak{g})\right)$ for some integer $n$. For every module $M \in \mathrm{Ob}(\mathcal{O})$ one has

$$
E \otimes\left(U(\mathfrak{g}) /|\lambda|^{n} U(\mathfrak{g})\right) \bigotimes_{U(\mathfrak{g})}^{\tau} M=E \otimes\left(U(\mathfrak{g}) /|\lambda|^{n} U(\mathfrak{g})\right)^{\tau} \bigotimes_{U(\mathfrak{g})} M
$$

thus, to prove the Lemma it is enough to construct natural isomorphisms of Harish-Chandra bimodules

$$
\phi_{E}: E \otimes\left(U(\mathfrak{g}) /|\lambda|^{n} U(\mathfrak{g})\right) \cong{ }^{\tau}\left(E \otimes\left(U(\mathfrak{g}) /|\lambda|^{n} U(\mathfrak{g})\right)\right)^{\tau}
$$

for all finite dimensional $\mathfrak{g}$-modules $E$.

For this we will use functor $\mathbb{V}: \mathcal{H} \rightarrow Z(\mathfrak{g}) \otimes Z(\mathfrak{g})-\bmod$ constructed by Soergel in [S92]. For a Harish-Chandra bimodule $M$ let $\mathbb{V}_{n}(M)=\operatorname{Hom}_{\mathcal{H}}\left(P_{n}, M\right)$, and $\mathbb{V}_{n}^{\tau}(M)=\operatorname{Hom}_{\mathcal{H}}\left(\tau\left(P_{n}\right)^{\tau}, M\right)$ where $P^{n}$ is a projective cover of simple principle series $L$ in $\mathcal{H}_{|\lambda|^{n}}$. Since central elements are stable under the automorphism of $\mathfrak{g}$ corresponding to $s_{\alpha}$, we have an equality $\mathbb{V}^{\tau}(M)=\mathbb{V}\left({ }^{\tau} M^{\tau}\right)$ of $Z(\mathfrak{g}) \otimes Z(\mathfrak{g})$-modules for every $M \in \mathcal{H}$. Choose an isomorphism $P_{n} \cong{ }^{\tau} P_{n}^{\tau}$. It defines an isomorphism of functors $\tilde{\phi}: \mathbb{V}_{n} \rightarrow \mathbb{V}_{n}^{\tau}$. Let $\tilde{\phi}^{E}$ be the evaluation of $\tilde{\phi}$ on $E \otimes\left(U(\mathfrak{g}) /|\lambda|^{n} U(\mathfrak{g})\right)$.

By [S92, Theorem 13], for a projective modules $P, Q$ in $\mathcal{H}_{|\lambda|^{n}}$ one has a natural isomorphism $\operatorname{Hom}_{\mathcal{H}}(P, Q) \cong \operatorname{Hom}_{Z(\mathfrak{g}) \otimes Z(\mathfrak{g})}\left(\mathbb{V}_{n}(P), \mathbb{V}_{n}(Q)\right)$. Similarly for $\mathbb{V}_{n}^{\tau}$. Define $\phi_{E}$ to be the unique isomorphism such that the following diagram is commutative

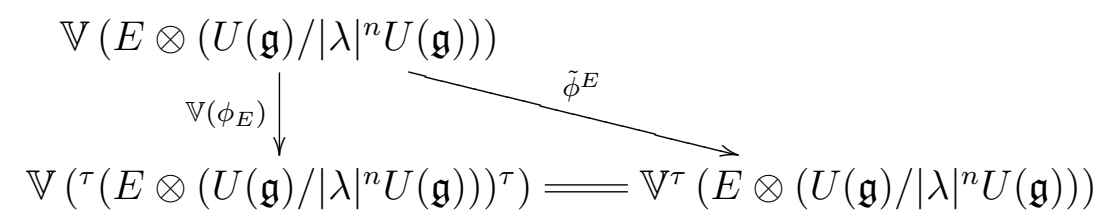

The naturality of $\phi_{E}$ follows from the naturality of $\tilde{\phi}^{E}$. 
From the discussion above it follows that Arkhipov's functors naturally commute with projective functors. These functors are right exact thus they are defined up to isomorphism by their values on $M(\lambda)$.

5.6. Relations between functors on $\mathcal{O}$. Now we are ready to present some relations between the above functors.

Proposition 6. Let $S$ be a subset of simple roots of $\mathfrak{g}$ and $w_{S}$ be the longest element in the Weyl group $W_{S}$, then $\mathcal{L}_{\ell\left(w_{S}\right)} \mathcal{A}_{w_{S}} \cong \Gamma_{S}$ and $\mathcal{R}_{\ell\left(w_{S}\right)} \mathcal{C}_{w_{S}}^{J} \cong * \circ \Gamma_{S} \circ *$.

Proof. By [AnS, Theorem 2.2] $\mathcal{L}_{i} \mathcal{A}_{s}=0$ for any integer $i>1$ and a simple reflection $s \in W$. By [AnS, (2.3)] for regular $\lambda \in \mathfrak{h}^{*}$ such that $s \cdot \lambda<\lambda$ one has $\mathcal{A}_{s}(M(\lambda))=M(s \cdot \lambda)$. Using [AnS, Theorem 2.2] one deduces that for $w=w_{1} s$ with $s$ being simple reflection and $l(w)=$ $l\left(w_{1}\right)+1$ the functor $\mathcal{A}_{s}$ maps projective modules into $\mathcal{A}_{w_{1}}$-acyclic. Thus by induction from Grothendieck spectral sequence we get that $\mathcal{L}_{i} \mathcal{A}_{w}=0$ for any $w \in W$ and $i>\ell(w)$ and $\mathcal{L}_{i} \mathcal{A}_{w}=\mathcal{L}_{1} \mathcal{A}_{s_{1}} \circ \cdots \circ \mathcal{L}_{1} \mathcal{A}_{s_{n}}$ for any reduced decomposition $w=s_{1} \ldots s_{n}$. From the long exact cohomology sequence it follows that $\mathcal{L}_{\ell(w)} \mathcal{A}_{w}$ are left exact for any $w \in W$. These functors naturally commute with projective functors by Lemma 1, thus they are defined up to an isomorphism by their values on $M(\lambda)^{*}$. By [AnS, Theorem 2.3], for $\alpha \in \pi$ the module $\mathcal{L}_{1} \mathcal{A}_{s_{\alpha}}\left(M(\lambda)^{*}\right)$ is the kernel of the unique nontrivial map $M(\lambda)^{*} \rightarrow M\left(s_{\alpha} \lambda\right)^{*}$ which is the maximal $\mathfrak{g}_{-\alpha}$-finite submodule of $M(\lambda)^{*}$. Thus it is isomorphic to $\Gamma_{\{\alpha\}}\left(M(\lambda)^{*}\right)$. The first statement now follows from the Theorem 5 by an easy induction on the cardinality of $S$, the second isomorphism can be proved by dual arguments.

Note that we can easily reprove the braid relations for Arkhipov's functors and Joseph's version of Enright's completion functors just by computing the values of corresponding compositions of functors on $M(\lambda)$ and $M(\lambda)^{*}$ respectively. Similarly we can reprove a result of Mazorchuk and the author (see KM1) that $\mathcal{A}_{\alpha} \cong * \circ \mathcal{C}_{\alpha}^{J} \circ *$ and more or less well known facts that $\mathcal{C}_{\alpha}^{J} \circ \mathcal{C}_{\alpha}^{J} \cong \mathcal{C}_{\alpha}^{D}$ and $\left(\mathcal{C}_{\alpha}^{J}\right)^{3} \cong\left(\mathcal{C}_{\alpha}^{J}\right)^{2}$.

Proposition 7. Suppose $\lambda \in \mathfrak{h}^{*}$ is integral dominant regular, for $\alpha \in \pi$ let $\lambda_{\alpha} \in \lambda+\mathcal{P}(R)$ be dominant such that $W_{\lambda_{\alpha}}=\left\{e, s_{\alpha}\right\}$. Then

$$
\begin{aligned}
& \mathcal{C}_{\alpha}^{D} \cong \mathcal{C}_{\alpha}^{J} \circ \mathcal{C}_{\alpha}^{J} \\
& \left(\mathcal{C}_{\alpha}^{J}\right)^{3} \cong\left(\mathcal{C}_{\alpha}^{J}\right)^{2} \\
& \left(\mathcal{A}_{\alpha}\right)^{3} \cong\left(\mathcal{A}_{\alpha}\right)^{2} \\
& \Gamma_{\alpha} \cong \mathcal{L}_{1} \mathcal{A}_{\alpha}
\end{aligned}
$$$$
\begin{aligned}
& \mathcal{C}_{\alpha}^{D} \cong \mathcal{C}_{M\left(\lambda_{\alpha}\right)^{*}} \\
& \mathcal{A}_{\alpha} \cong * \circ \mathcal{C}_{\alpha}^{J} \circ * \\
& \left(\mathcal{A}_{\alpha}\right)^{2} \cong \tilde{\mathcal{C}}_{M\left(\lambda_{\alpha}\right)} \\
& \mathcal{R}_{1} \mathcal{C}_{\alpha}^{J} \cong * \circ \Gamma_{\alpha} \circ *
\end{aligned}
$$ 
Proof. All these isomorphisms can be proved just by computing the value of both sides on projective Verma module resp. its dual similarly to the proof of Proposition 6

Using Proposition 1 one can easily get some non-trivial natural transformation between the above functors just by computing them on $M(\lambda)$ resp. $M(\lambda)^{*}$.

Lemma 7. Let $w \in W, S \subset \pi$ and $\lambda \in \mathfrak{h}^{*}$ be integral and regular. There exist nontrivial morphisms between the following functors on $\mathcal{O}_{|\lambda|}$ :

$$
\begin{aligned}
& \text { - } \operatorname{Id}_{\mathcal{O}_{|\lambda|}} \rightarrow \mathcal{C}_{w}^{J} \\
& \text { - } \mathcal{A}_{w} \rightarrow \operatorname{Id}_{\mathcal{O}_{|\lambda|}} \\
& \text { - } \mathcal{C}_{\pi}^{D} \rightarrow \operatorname{Id}_{\mathcal{O}_{|\lambda|}} \\
& \text { - } \mathcal{C}_{\pi}^{D} \rightarrow \Gamma_{S}
\end{aligned}
$$

5.7. Parabolic category $\mathcal{O}$. Let $\mathfrak{p}$ and $\mathfrak{m}$ be as in Section 3 and $\mathcal{O}^{\mathfrak{p}}$ be the parabolic category $\mathcal{O}$. By definition, it is a full subcategory of $\mathcal{O}$ whose objects are locally $\mathfrak{m}$-finite $\mathfrak{g}$-modules.

The functors $\Gamma_{\mathfrak{p}}$ and $* \circ \Gamma_{\mathfrak{p}} \circ *$ can be viewed as functors from $\mathcal{O}$ to $\mathcal{O}^{\mathfrak{p}}$. In this case they are right resp. left adjoint to the inclusion functor $\mathcal{O}^{\mathfrak{p}} \rightarrow \mathcal{O}$. In particular this implies that if $P$ is a projective module in $\mathcal{O}$ and $I$ is an injective module in $\mathcal{O}$ then $\left(\Gamma_{\mathfrak{p}}\left(P^{*}\right)\right)^{*}$ is projective and $\Gamma_{\mathfrak{p}}(I)$ is injective in $\mathcal{O}^{\mathfrak{p}}$. Moreover $\mathcal{O}^{\mathfrak{p}}$ has enough projective resp. injective objects and every projective resp injective object is the image of a projective resp. an injective object in $\mathcal{O}$ under $* \circ \Gamma_{\mathfrak{p}} \circ *$ resp. $\Gamma_{\mathfrak{p}}$. Let $\mathcal{O}_{i n t}^{\mathfrak{p}}=\mathcal{O}^{\mathfrak{p}} \cap \mathcal{O}_{\text {int }}$. The following statement was suggested to the author by C. Stroppel.

Proposition 8. Let $\lambda$ be a dominant regular integral weight. The parabolic category $\mathcal{O}_{\text {int }}^{\mathfrak{p}}$ together with the dominant object $\left(\Gamma_{\mathfrak{p}}\left(M(\lambda)^{*}\right)\right)^{*}$ and restrictions of projective functors on $\mathcal{O}_{\text {int }}$ to $\mathcal{O}_{\text {int }}^{\mathfrak{p}}$ is a category with full projective functors.

Proof. Again the Conditions (PF1), (PF2) and (PF3) are obvious. To prove (PF4) take two finite dimensional modules $E$ and $F$ and a morphism $f: E \otimes\left(\Gamma_{\mathfrak{p}}\left(M(\lambda)^{*}\right)\right)^{*} \rightarrow F \otimes\left(\Gamma_{\mathfrak{p}}\left(M(\lambda)^{*}\right)\right)^{*}$. The morphism $f$ can be lifted to a morphism $\hat{f}: E \otimes M(\lambda) \rightarrow F \otimes M(\lambda)$ between projective covers of $\left(\Gamma_{\mathfrak{p}}\left(M(\lambda)^{*}\right)\right)^{*}$ resp. $\left(\Gamma_{\mathfrak{p}}\left(M(\lambda)^{*}\right)\right)^{*}$ in $\mathcal{O}$. By Proposition $4 \hat{f}$ comes from a natural transformation $\tilde{f}: E \otimes \operatorname{Pr}_{\lambda}\left({ }_{-}\right) \rightarrow F \otimes \operatorname{Pr}_{\lambda}\left(_{-}\right)$. The proposition now follows from the observation that every natural transformation $E \otimes P r_{\lambda}(-) \rightarrow F \otimes P r_{\lambda}\left({ }_{-}\right)$of functors on $\mathcal{O}$ is by restriction a natural transformation of functors on $\mathcal{O}^{\mathfrak{p}}$. 
Remark 3. Another way to prove the above Proposition is to note that $\mathcal{O}_{\text {int }}^{\mathfrak{p}}=\operatorname{coker}\left(\mathcal{F}(\mathfrak{g}) \otimes\left(\Gamma_{\mathfrak{p}}\left(M(\lambda)^{*}\right)\right)^{*}\right)$ and apply Proposition 3 . This gives also a description of quasidominant objects of $\mathcal{O}_{i n t}^{\mathfrak{p}}$.

\section{Categorical Realization of Arkhipov's and Joseph's FUNCTORS}

In this section we present a categorical realization of Arkhipov's and Joseph's functors from [KM1]. Let $A$ be a finite dimensional associative algebra and $\Lambda$ the set of isomorphism classes of simple $A$-modules. The simple $A$-module $L(\lambda), \lambda \in \Lambda$, has the projective cover $P(\lambda)$ and the injective envelope $I(\lambda)$.

Let $\Upsilon$ be a subset of $\Lambda$. We denote by $\mathfrak{d}_{\Upsilon}(-)$ the functor, which is defined on $A$-module $M$ as follows. First we realize $M$ as a submodule of its injective envelope $I_{M}$ say, that is we get a monomorphism $\mathfrak{z}$ : $M \rightarrow I_{M}$. Now we can compute the maximal coextension $M^{1}$ of $M$ with non- $\Upsilon$ composition subquotients (the intersection of the kernels of all possible maps from $I_{M}$ to $\Upsilon$-injectives, which annihilate $M$ ). Now $\mathfrak{d}_{\Upsilon}(M)$ is, by definition, the quotient of $M^{1}$ by maximal submodule, which does not contain simple subquotients of type $\Upsilon$. Let $f: M \rightarrow N$ be a homomorphism, then one can lift $f$ to a map, $\hat{f}: M^{1} \rightarrow N^{1}$ in a non-unique way but up to the choice of a map from $M^{1} / M$ to $N^{1}$. In fact, since all composition subquotients of $M^{1} / M$ are not of type $\Upsilon$, the image of any map from $M^{1} / M$ to $N^{1}$ is contained in the maximal submodule of $N^{1}$, which does not have any composition subquotients of type $\Upsilon$. The latter one is then killed when taking the quotient of $N^{1}$ by maximal submodule, which does not contain simple subquotients of type $\Upsilon$. Thus above procedure defines a functor from $A$-mod to $A$-mod. We will call $\mathfrak{d}_{\Upsilon}(-)$ the functor of a partial approximation with respect to $\Upsilon$.

It is clear that the functor $\mathfrak{d}_{\Upsilon}$ comes together with a natural map $\mathfrak{d}_{\Upsilon}^{\text {nat }}: M \rightarrow \mathfrak{d}_{\Upsilon}(M)$. This map is a composition of $\mathfrak{z}: M \rightarrow I_{M}$, whose image lies inside $M^{1}$, followed by the natural projection of $M^{1}$ onto $\mathfrak{d}_{\Upsilon}(M)$. Moreover, it is easy to see that the kernel of the natural map $M \rightarrow \mathfrak{d}_{\Upsilon}(M)$ coincides with the maximal submodule of $M$, which does not have subquotients of type $\Upsilon$. If the module $M$ is injective, its coextension coincides with $M$ and thus the natural map $M \rightarrow \mathfrak{d}_{\Upsilon}(M)$ is surjective.

Using the dual construction one defines the functor $\tilde{\mathfrak{d}}_{\Upsilon}$ of a partial coapproximation with respect to $\Upsilon$. For every module $M$ one gets a natural morphism $\tilde{\mathfrak{d}}_{\Upsilon}^{\text {nat }}: \tilde{\mathfrak{d}}_{\Upsilon}(M) \rightarrow M$. Its properties are dual to those of $\mathfrak{d}_{\Upsilon}$. In particular, for all $M$ the cokernel of this morphism is the 
maximal quotient of $M$, which does not have subquotients of type $\Upsilon$. Further, $\tilde{\mathfrak{d}}_{\Upsilon}^{\text {nat }}$ is injective for projective $M$. If $A$ has a simple preserving duality, $D$ say, then $\tilde{\mathfrak{d}}_{\Upsilon} \simeq D \circ \mathfrak{d}_{\Upsilon} \circ D$.

Lemma 8. The functor $\tilde{\mathfrak{d}}_{\Upsilon}: A-\bmod \rightarrow A-\bmod$ is left adjoint to the functor $\mathfrak{d}_{\Upsilon}: A-\bmod \rightarrow A$-mod.

Proof. Let $M, N \in A$-mod and $f: \tilde{\mathfrak{d}}_{\Upsilon}(M) \rightarrow N$ be a homomorphism. We construct the diagram

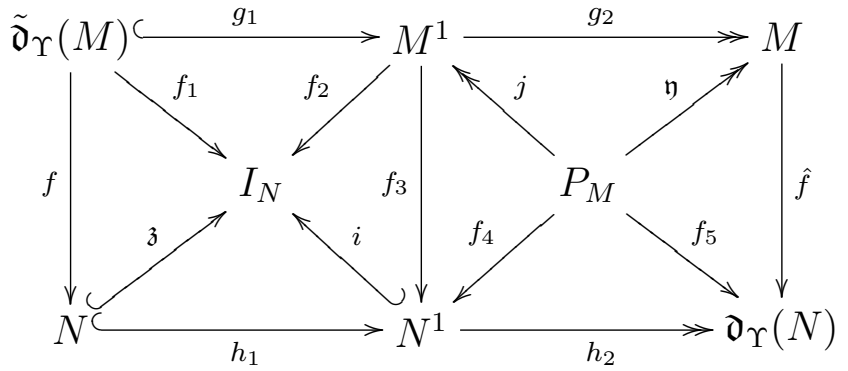

in the following way: the module $N^{1}$ (resp. $M^{1}$ ) is the maximal coextension (resp. extension) of $N$ (resp. $M$ ) with subquotients, which are not of type $\Upsilon$. From the definition of $N^{1}$ and $M^{1}$ we have the maps $h_{1}: N \rightarrow N^{1}, i: N^{1} \rightarrow I_{N}$ such that $i \circ h_{1}=\mathfrak{z}_{N}$, and the maps $g_{2}: M^{1} \rightarrow M, j: P_{M} \rightarrow M^{1}$ such that $g_{2} \circ j=\mathfrak{y}_{M}$. From the definition of $\tilde{\mathfrak{d}}_{\Upsilon}$ and $\mathfrak{d}_{\Upsilon}$ we also have injection $g_{1}: \tilde{\mathfrak{d}}_{\Upsilon}(M) \rightarrow M^{1}$ and surjection $h_{2}: N^{1} \rightarrow \mathfrak{d}_{\Upsilon}(N)$ such that $g_{2} \circ g_{1}=\tilde{\mathfrak{d}}_{\Upsilon}^{\text {nat }}$ and $h_{2} \circ h_{1}=\mathfrak{d}_{\Upsilon}^{\text {nat }}$ respectively.

Now we proceed to the construction of the maps $f_{i}, i=1,2, \ldots, 5$, and $\hat{f}$. Set $f_{1}=\mathfrak{z}_{N} \circ f$. Since $g_{1}$ is an injective map and $I_{N}$ is an injective module, there exists $f_{2}: M \rightarrow I_{N}$, making the corresponding triangle commutative. We remark that $f_{2}$ is not unique in general, but is defined only up to the maps from $\operatorname{coker}\left(g_{1}\right)$ to $I_{N}$. Since all simple subquotients of coker $\left(g_{1}\right)$ are not of type $\Upsilon$ and $N^{1}$ is the maximal coextension of $N$ with such subquotients, the image of $f_{2}$ belongs to $N^{1}$, giving us the map $f_{3}: M^{1} \rightarrow N^{1}$. The map $f_{3}$ depends on the choice of $f_{2}$ and thus is not uniquely defined by $f$. However, since the socle of $\mathfrak{d}_{\Upsilon}(N)$ consists only of simples, which are of type $\Upsilon$, the composition $h_{2} \circ f_{3}$ is in fact independent of the choice of $f_{2}$ and hence is uniquely determined by $f$. We define $f_{4}=f_{3} \circ j$ and $f_{5}=h_{2} \circ f_{4}$. We have $\operatorname{ker}(j) \subset \operatorname{ker}\left(f_{5}\right)$ by construction. Further, $\operatorname{ker}\left(g_{2}\right)$ contains only simple subquotients of type $\Upsilon$, and the socle of $\mathfrak{d}_{\Upsilon}(N)$ does not contain such subquotients. This implies $\operatorname{ker}\left(\mathfrak{y}_{M}\right) \subset \operatorname{ker}\left(f_{5}\right)$ and thus there exists unique $\hat{f}$, which finally makes the whole diagram commutative. The commutativity of the diagram and the fact that $h_{2} \circ f_{3}$ does not depend on the choice of $f_{2}$, 
implies that $\hat{f}$ is uniquely determined by $f$. Now one easily checks that the dual construction sends $\hat{f}$ back to $f$, thus providing the necessary isomorphism $\operatorname{Hom}_{A}\left(\tilde{\mathfrak{d}}_{\Upsilon}(M), N\right)=\operatorname{Hom}_{A}\left(M, \mathfrak{d}_{\Upsilon}(N)\right)$. Naturality of this isomorphism follows from the construction.

Corollary 3. The functor $\tilde{\mathfrak{d}}_{\Upsilon}: A-\bmod \rightarrow A-\bmod$ is right exact and the functor $\mathfrak{d}_{\Upsilon}: A-\bmod \rightarrow A-\bmod$ is left exact.

For $\alpha \in \pi$ let $\Upsilon_{\alpha}$ be the set of isoclasses of all simple $X_{-\alpha}$-free objects of $\mathcal{O}_{\text {int }}$, and let $\mathfrak{d}_{\alpha}$ (resp. $\left.\tilde{\mathfrak{d}}_{\alpha}\right)$ denote the functors $\mathfrak{d}_{\Upsilon_{\alpha}}\left(\right.$ resp. $\left.\tilde{\mathfrak{d}}_{\Upsilon_{\alpha}}\right)$ on $\mathcal{O}_{\text {int }}$.

Lemma 9. Let $E$ be a finite dimensional $\mathfrak{g}$-module. The functors $\mathfrak{d}_{\alpha}$ and $\tilde{\mathfrak{d}}_{\alpha}$ from $\mathcal{O}_{\text {int }}$ to $\mathcal{O}_{\text {int }}$ naturally commute with $E \otimes_{-}$.

Proof. Both functors $\mathfrak{d}_{\alpha}\left(E \otimes_{-}\right)$and $E \otimes \mathfrak{d}_{\alpha}\left({ }_{-}\right)$are left exact as compositions of left exact $\mathfrak{d}_{\alpha}$ with exact $E \otimes_{-}$. We also have natural transformations $(E \otimes i d) \circ \mathfrak{d}_{\alpha}^{n a t}: E \otimes_{-} \rightarrow E \otimes \mathfrak{d}_{\alpha}\left({ }_{-}\right)$and $\mathfrak{d}_{\alpha}^{n a t} \circ(E \otimes i d)$ : $E \otimes_{-} \rightarrow \mathfrak{d}_{\alpha}\left(E \otimes_{-}\right)$.

Let $M$ be an injective module and $N$ be the maximal submodule of $M$, which contains only subquotients not of type $\Upsilon_{\alpha}$, or, equivalently, the maximal $X_{-\alpha}$-locally finite submodule of $M$. As $M$ is injective, there is a short exact sequence

$$
0 \rightarrow N \rightarrow M \stackrel{\mathfrak{d}_{\alpha}^{n a t}}{\rightarrow} \mathfrak{d}_{\alpha}(M) \rightarrow 0
$$

Applying the exact functor $E \otimes_{-}$we get the short exact sequence

$$
0 \rightarrow E \otimes N \rightarrow E \otimes M \stackrel{\mathbf{I d} \otimes \mathfrak{d}_{\alpha}^{n a t}}{\longrightarrow} E \otimes \mathfrak{d}_{\alpha}(M) \rightarrow 0
$$

and the module $E \otimes N$ is the maximal $X_{-\alpha}$-locally finite submodule of $E \otimes M$.

On the other hand $E \otimes M$ is injective and we have the following short exact sequence:

$$
0 \rightarrow K \rightarrow E \otimes M \stackrel{\mathfrak{d}_{\alpha}^{n a t}}{\rightarrow} \mathfrak{d}_{\alpha}(E \otimes M) \rightarrow 0
$$

where $K$ is the maximal $X_{-\alpha}$-locally finite submodule of $E \otimes M$. Hence $K=E \otimes N$ and we get the isomorphism

$$
\mathfrak{i}_{M}: E \otimes \mathfrak{d}_{\alpha}(M) \rightarrow \mathfrak{d}_{\alpha}(E \otimes M) .
$$

Moreover $\operatorname{ker}\left(\mathbf{I} \mathbf{d} \otimes \mathfrak{d}_{\alpha}^{n a t}(M)\right)=\operatorname{ker}\left(\mathfrak{d}_{\alpha}^{n a t}(\mathbf{I} \mathbf{d} \otimes M)\right)=E \otimes N$. Suppose we are given another injective module $M^{\prime}$ and a morphism $f: M \rightarrow M^{\prime}$. 
Consider the following diagram

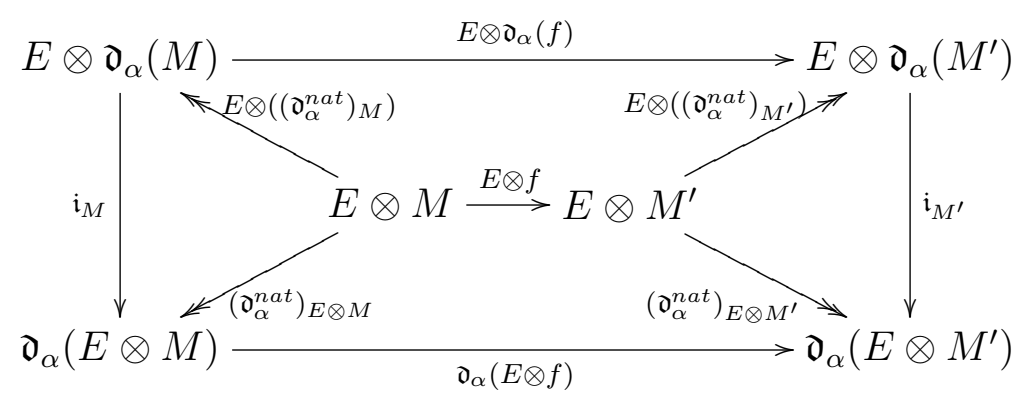

Right and left triangles in this diagram commute by construction. Both small quadrangles commute by naturality of $\mathfrak{d}_{\alpha}^{\text {nat }}$. So the surjectivity of $\left(\mathfrak{d}_{\alpha}^{n a t}\right)_{M}$ and $\left(\mathfrak{d}_{\alpha}^{n a t}\right)_{M^{\prime}}$ implies the commutativity of the big quadrangle. Thus, by standard arguments, the collection of isomorphisms $\mathfrak{i}_{M}$ for injective $M$ can be extended to isomorphism $\mathfrak{d}_{\alpha}\left(E \otimes_{-}\right) \cong E \otimes \mathfrak{d}_{\alpha}\left({ }_{-}\right)$.

To show that $\mathfrak{d}_{\alpha}$ naturally commutes with $E \otimes_{-}$take a morphism of functors $\mathfrak{f}: E \otimes_{-} \rightarrow E \otimes_{-}$. The following diagram with exact rows commutes by naturality of $\mathfrak{f}$.

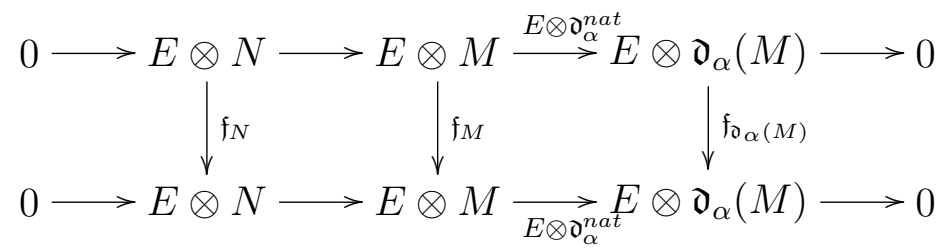

The following diagram with exact rows commutes by naturality of $\mathfrak{d}_{\alpha}^{\text {nat }}$.

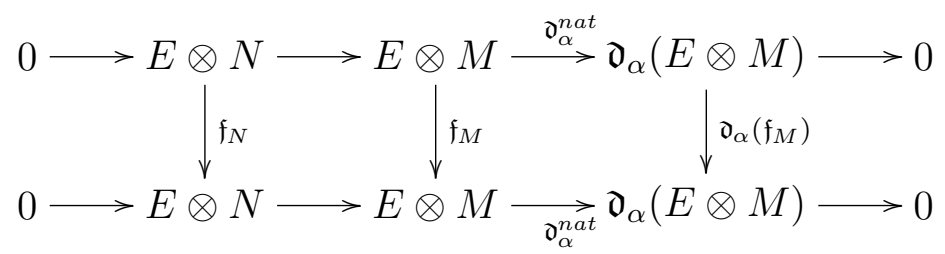

Gluing both diagrams together one gets the required statement for injective modules. The extension to arbitrary modules is the technicality which we omit here. The statement for $\tilde{\mathfrak{d}}_{\alpha}$ is obtained by dual arguments.

The next theorem provides a categorical description of Enright's and Joseph's functors.

Theorem 7. $\mathcal{C}_{\alpha}^{J} \cong \mathfrak{d}_{\alpha}$ and $\mathcal{A}_{\alpha} \cong \tilde{\mathfrak{d}}_{\alpha}$.

Proof. Using Theorem 5 and Lemma 9, one reduces the proof to the computation of left and right hand side on dominant Verma module resp. its dual. 


\section{Harish-Chandra Bimodules}

Let $\chi$ be a maximal ideal in $Z(\mathfrak{g})$ fixed through this section. We assume that $\chi$ is regular. Neither category $\mathcal{H}$ nor $\mathcal{H}_{\chi}^{\infty}$ have enough projective objects. To be able to deal with these categories in our framework we have to consider a slightly biger category (this is a slight modification of the construction in [BeGi]).

An $U(\mathfrak{g})$-module $M$ will be called complete (with respect to $\chi$-adic topology) if $M=\lim _{n}\left(M / \chi^{n} M\right)$. Let $\widehat{U}_{\chi}=\varliminf_{n}\left(U(\mathfrak{g}) / U(\mathfrak{g}) \chi^{n}\right)$ be the completion of $U(\mathfrak{g})$. The algebra $\widehat{U}_{\chi}$ is a complete $U(\mathfrak{g})$ module and it is Noetherian because $U(\mathfrak{g})$ is. Thus, by Artin-Rees lemma, any finite generated $\widehat{U}_{\chi}$-module is complete. In particular finite generated $\widehat{U}_{\chi}$-modules form an abelian category. Any complete $U(\mathfrak{g})$-module has a natural structure of $\widehat{U}_{\chi}$-module.

Let $\widehat{\mathcal{H}}_{\chi}$ be a full subcategory of the category of $U(\mathfrak{g})$-bimodules whose objects are all $U(\mathfrak{g})$-bimodules $X$ such that $X$ is complete as right $U(\mathfrak{g})$ module, $X$ is finite generated as right $\widehat{U}_{\chi}$-module and the adjoint action of $\mathfrak{g}$ on $X / X \chi^{n}$ is locally finite for any positive integer $n$. Clearly every object of $\widehat{\mathcal{H}}_{\chi}$ has a canonical structure of right $\widehat{U}_{\chi}$-module and every object of $\mathcal{H}_{\chi}^{\infty}$ is an object of $\widehat{\mathcal{H}}_{\chi}$ in a canonical way. An $U(\mathfrak{g})$-bimodule $M$ is an object of $\widehat{\mathcal{H}}_{\chi}$ if and only if $M$ is finitely generated as right $\widehat{U}_{\chi}$-module and $M=\lim _{n} M / M \chi^{n}$ with $M / M \chi^{n} \in \mathrm{Ob}(\mathcal{H})$.

For any positive integer $n$ and finite dimensional module $F$ the object $F \otimes U(\mathfrak{g}) / U(\mathfrak{g}) \chi^{n}$ is a projective object in the category $\mathcal{H}_{\chi^{n}}$ (this follows from Soergel's description of $\mathcal{H}_{\chi^{n}}$ as "thick" category $\mathcal{O}$, see [S86]). The next lemma provides an analogous statement for the completed category $\widehat{\mathcal{H}}_{\chi}$.

Lemma 10. For every left finite dimensional $\mathfrak{g}$-module $F$ the $U(\mathfrak{g})$ bimodule $F \otimes \widehat{U}_{\chi}$ is projective object of $\widehat{\mathcal{H}}_{\chi}$. The category $\widehat{\mathcal{H}}_{\chi}$ has enough projective objects.

Proof. First note that $F \otimes \widehat{U}_{\chi}=\lim _{n} F \otimes\left(U(\mathfrak{g}) / U(\mathfrak{g}) \chi^{n}\right)$, thus $F \otimes \widehat{U}_{\chi}$ is an object of $\widehat{\mathcal{H}}_{\chi}$.

Suppose we are given two objects $L, M$ in $\widehat{\mathcal{H}}_{\chi}$, an epimorphism $\pi: M \rightarrow L$ and a morphism $\psi: \widehat{U}_{\chi} \rightarrow L$. The subspace $\mathbb{C} 1 \subset \widehat{U}_{\chi}$ is a trivial $\mathfrak{g}$-module with respect to the adjoint action, thus $\psi(\mathbb{C} 1) \subset L$ is a 1-dimensional $\mathfrak{g}$-submodule of $L$ (with respect to the adjoint action). Since $\pi$ is surjective and $M / M \chi^{n}$ is a direct sum of finite dimensional $\mathfrak{g}$-modules there exist a non-zero element $v \in \pi^{-1}(\psi(1))$ such that $\mathbb{C} v$ is invariant under the adjoint action of $\mathfrak{g}$. Define a map $\phi: \widehat{U}_{\chi} \rightarrow M$ 
by $u \mapsto v u$. Obviously it is a morphism of right $\widehat{U}_{\chi}$-modules. Since $\mathbb{C} v$ is invariant under the adjoint action the map $\phi$ is a morphism of $U(\mathfrak{g})$-bimodules. By the construction $\pi \circ \phi=\psi$. This proves that $\widehat{U}_{\chi}$ is projective, thus so are $F \otimes \widehat{U}_{\chi}$ for arbitrary finite dimensional $\mathfrak{g}$-module $F$.

The simple objects of $\widehat{\mathcal{H}}_{\chi}$ are the simple objects of its full subcategory $\mathcal{H}_{\chi}$. Every simple module in $\mathcal{H}_{\chi}$ can be covered by $F \otimes(U(\mathfrak{g}) / \chi U(\mathfrak{g}))$ for suitable finite dimensional $\mathfrak{g}$-module $F$. This implies the second statement of the lemma.

The category $\widehat{\mathcal{H}}_{\chi}$ possesses a block decomposition

$$
\widehat{\mathcal{H}}_{\chi}=\bigoplus_{\zeta \in \operatorname{Max} Z(\mathfrak{g})}{ }_{\zeta}^{\infty} \widehat{\mathcal{H}}_{\chi}
$$

where the objects of ${ }_{\zeta}^{\infty} \widehat{\mathcal{H}}_{\chi}$ are projective limits of objects of ${ }_{\zeta}^{\infty} \mathcal{H}_{\chi^{n}}$. Let $\operatorname{Pr}_{\zeta}$ denotes the projection functor onto the block ${ }_{\zeta}^{\infty} \mathcal{H}_{\chi^{n}}$. Any bimodule $M \in \mathrm{Ob}\left(\chi_{\chi}^{\infty} \widehat{\mathcal{H}}_{\chi}\right)$ has a natural structure of left $\widehat{U}_{\chi}$-module. Moreover for any bimodule $N$ in $\widehat{\mathcal{H}}_{\chi}$ the bimodule $N \bigotimes_{\widehat{U}_{\chi}} M$ is again an object of $\widehat{\mathcal{H}}_{\chi}$.

Theorem 8. (i) The category $\widehat{\mathcal{H}}_{\chi}$ together with the dominant object $\widehat{U}_{\chi}$ and all direct summands of the functors

$$
\left\{E \otimes \operatorname{Pr}_{\chi}(-) \mid E \in \mathcal{F}(\mathfrak{g})\right\} \cup\{\mathbf{I d}\}
$$

is a category with full projective functors.

(ii) $M \in \mathrm{Ob}\left(\widehat{\mathcal{H}}_{\chi}\right)$ is quasidominant if and only if $M \in \mathrm{Ob}\left(\begin{array}{l}\infty \\ \chi\end{array} \widehat{\mathcal{H}}_{\chi}\right)$.

(iii) Every right exact additive endofunctor of $\widehat{\mathcal{H}}_{\chi}$ which naturally commutes with projective functors is isomorphic to $-\bigotimes_{\widehat{U}_{\chi}} M$ for some bimodule $M$ in $\underset{\chi}{\infty} \widehat{\mathcal{H}}_{\chi}$.

Proof. It is sufficient to consider only the case of tensoring with finite dimensional modules. Condition (PF1) is tautological. Conditions (PF2) and (PF3) follow from Lemma 10. For every finitedimensional $\mathfrak{g}$-module $E$ there is an isomorphism of functors $E \otimes$ $\operatorname{Pr}_{\chi}(-) \cong\left(E \otimes \widehat{U}_{\chi}\right) \otimes_{\widehat{U}_{\chi}} \operatorname{Pr}_{\chi}(-)$. This provides the Condition (PF4). The remaining statements are now easy.

Let $n$ be a positive integer. Restricting the above theorem to the full subcategory of $\widehat{\mathcal{H}}_{\chi}$ whose objects are all modules annihilated by $\chi^{n}$ from the right, we easily get the following corollary generalizing Proposition 4. 
Corollary 4. (i) The category $\mathcal{H}_{\chi^{n}}$ together with the dominant $o b$ ject $U(\mathfrak{g}) / U(\mathfrak{g}) \chi^{n}$ and all direct summands of functors

$$
\left\{E \otimes \operatorname{Pr}_{\chi}(-) \mid E \in \mathcal{F}(\mathfrak{g})\right\} \cup\{\mathbf{I d}\}
$$

is a category with full projective functors.

(ii) $M \in \mathrm{Ob}\left(\mathcal{H}_{\chi^{n}}\right)$ is quasidominant if and only if $M \in \mathrm{Ob}\left(\chi^{n} \mathcal{H}_{\chi^{n}}\right)$.

(iii) Every right exact additive endofunctor of $\mathcal{H}_{\chi^{n}}$ which naturally commutes with projective functors is isomorphic to $-\bigotimes_{U(\mathfrak{g})} M$ for some bimodule $M$ in $\chi^{n} \mathcal{H}_{\chi^{n}}$.

Proof. The first and the third statements immediately follow from Theorem 8. The second statement follows from the observation that multiplication with central elements from the left is an endomorphism of the identity functor.

We have to remark, that projective objects of $\widehat{\mathcal{H}}_{\chi}$ can be identified with projective functors $F: \mathcal{M}_{\chi}^{\infty} \rightarrow \mathcal{M}_{Z f}$ in the sense of Definition 4 . By Theorem 8, the objects of $\widehat{\mathcal{H}}_{\chi}$ correspond then to the functors from $\mathcal{M}_{\chi}^{\infty}$ to $\mathcal{M}_{Z f}$ possessing a presentation by projective functors.

For a bimodule $M$ by $M_{\chi}^{\wedge}$ we denote the bimodule ${\underset{\varliminf}{\longleftarrow}}_{n} M / M \chi^{n}$. Similarly we define ${ }_{\chi}^{\wedge} M$. One can restrict Theorem 8 to the subcategory ${ }_{\chi}^{\infty} \widehat{\mathcal{H}}_{\chi}$ to obtain a structure of category with projective functors on it. The next theorem provides another structure of category with projective functors on ${ }_{\chi}^{\infty} \widehat{\mathcal{H}}_{\chi}$ induced by the tensoring with finite dimensional modules from the right.

Theorem 9. (i) The category ${ }_{\chi}^{\infty} \widehat{\mathcal{H}}_{\chi}$ together with the dominant object $\widehat{U}_{\chi}$ and all direct summand of the functors

$$
\left\{(-\otimes E)_{\chi}^{\wedge} \mid E \in \mathcal{F}(\mathfrak{g})\right\} \cup\{\mathbf{I d}\}
$$

is a category with full projective functors.

(ii) Every object of $\chi_{\chi}^{\infty} \widehat{\mathcal{H}}_{\chi}$ is quasi-dominant.

(iii) Every right exact additive endofunctor of ${ }_{\chi}^{\infty} \widehat{\mathcal{H}}_{\chi}$ which naturally commutes with projective functors is isomorphic to $M \bigotimes_{\widehat{U}_{\chi}}-$ for some bimodule $M$ in $\underset{\chi}{\infty} \widehat{\mathcal{H}}_{\chi}$.

Proof. Again, Condition (PF1) is tautological. The module $\widehat{U}_{\chi}$ is projective in ${ }_{\chi}^{\infty} \widehat{\mathcal{H}}_{\chi}$ by Lemma 10. Tensoring with finite dimensional modules maps projectives to projectives. This implies Condition (PF2).

For an object $M$ of $\widehat{\mathcal{H}}_{\chi}$ let ${ }^{s} M$ denotes the bimodule with transposed operation (see [Ja, 6.3]). It is clear that ${ }^{s}\left(\left(\widehat{U}_{\chi} \otimes E\right)_{\chi}^{\wedge}\right)={ }_{\chi}^{\wedge}\left(E \otimes \widehat{U}_{\chi}\right)$. The module ${ }_{\chi}^{\wedge}\left(E \otimes \widehat{U}_{\chi}\right)$ is projective in ${ }_{\chi}^{\infty} \widehat{\mathcal{H}}_{\chi}$, its top is canonically 
isomorphic to the top of $\operatorname{Pr}_{\chi}\left(E \otimes \widehat{U}_{\chi}\right)$. Every simple module in ${ }_{\chi}^{\infty} \widehat{\mathcal{H}}_{\chi}$ is isomorphic to $\mathcal{L}(M(\lambda), L(w \cdot \lambda))$ for some $w \in W$ (here $\lambda$ is a dominant weight such that $|\lambda|=\chi)$. Condition (PF3) now follows from [Ja, Satz 7.28] and Theorem 8.

Condition (PF4) and the remaining two statements of the theorem can be proved similarly to Theorem 8 .

Restricting to the subcategory of bimodules annihilated by $\chi^{n}$ from the right for some integer $n$ we get

Corollary 5. (i) The category ${ }_{\chi}^{\infty} \mathcal{H}_{\chi^{n}}$ together with the dominant object $U(\mathfrak{g}) / U(\mathfrak{g}) \chi^{n}$ and all direct summands of the functors

$$
\left\{(-\otimes E) /(-\otimes E) \chi^{n} \mid E \in \mathcal{F}(\mathfrak{g})\right\} \cup\{\mathbf{I d}\}
$$

is a category with full projective functors.

(ii) An object $M$ of ${ }_{\chi}^{\infty} \mathcal{H}_{\chi^{n}}$ is a quasidominant object if and only if $M \in \mathrm{Ob}\left({ }_{\chi^{n}} \mathcal{H}_{\chi^{n}}\right)$.

(iii) Every right exact additive endofunctor of $\mathcal{H}_{\chi^{n}}$ which naturally commutes with projective functors is isomorphic to $M \bigotimes_{U(\mathfrak{g})}$ - for some bimodule $M$ in ${ }_{\chi^{n}} \mathcal{H}_{\chi^{n}}$.

In particular, for $n=1$, we get another structure of a category with projective functors on the regular block $\mathcal{O}_{\chi}$ of the category $\mathcal{O}$. The projective functors in this case are only right exact. It is clear from the construction that the functors $\operatorname{Pr}_{\chi}\left(E \otimes_{-}\right)$on $\mathcal{O}$ naturally commute with such projective functors. In particular translation and wall crossing functors naturally commute with projective functors from Corollary 5 . Zuckerman's functors are the kernels of the natural transformations from the identity functor to the wall-crossing functors, shuffling functors are the cokernels of these natural transformations, thus they both also naturally commute with projective functors from Corollary 5. This structure of category with full projective functors on $\mathcal{O}_{\chi}$ can for example be used to establish relations between translation functors or the braid relations between the shuffling functors. (For this, using above theory, it is enough to check it on the dominant Verma module). It can also be applied to reprove the result of Bernstein and Gelfand [BG] that projective functors are determined up to isomorphism by their values on the dominant Verma module.

\section{Kostant's Problem}

In this section we show how the technique from Sections 2 and 4 combined with ideas, developed in [KM2], enable one to determine the 
algebra of adjoint finite endomorphisms of certain $\mathfrak{g}$-modules. Note that in $[\mathrm{Za}]$ this problem was solved for Whittaker modules.

One has a direct sum decomposition of $U(\mathfrak{g})$-bimodules

$$
\mathcal{L}(M, M)=\bigoplus_{\gamma \in \Gamma} \mathcal{L}(M, M)_{\gamma}
$$

where $\mathcal{L}(M, M)_{\gamma}$ is the maximal $U(\mathfrak{g})$-sub-bimodule of $\mathcal{L}(M, M)$ such that viewed as a $\mathfrak{g}$-module under the adjoint action it is isomorphic to a direct sum of simple finite dimensional modules whose highest weight belongs to $\gamma+\mathbb{Z} R$. Let $\Gamma=\mathcal{P}(R) / \mathbb{Z} R$ and let $\Gamma_{M} \subset \Gamma$ be a subgroup whose elements are all $\gamma \in \Gamma$ for which there exist a representative $\mu \in \mathcal{P}(R)$ with $\operatorname{Hom}_{\mathfrak{g}}\left(M, L(\mu+\rho)^{*} \otimes M\right) \neq 0$. For every $\mathfrak{g}$-module $N$ and for every finite dimensional module $F$ by [Ja, 6.8] one has

(4) $\operatorname{Hom}_{\mathfrak{g}}\left(F, \mathcal{L}(N, N)^{\mathrm{ad}}\right)=\operatorname{Hom}_{\mathfrak{g}}(F \otimes N, N)=\operatorname{Hom}_{\mathfrak{g}}\left(N, F^{*} \otimes N\right)$.

In particular this implies, that the direct sum in (3) runs over $\Gamma_{M}$.

Let $M$ be a $\mathfrak{g}$-module such that $\operatorname{Ann}_{U(\mathfrak{g})} M=U(\mathfrak{g})|\lambda|$ for some dominant $\lambda \in \mathfrak{h}^{*}$ and let $M^{\prime}$ be the translation of $M$ to the most singular character $\left|\mu^{\prime}\right|\left(\mu \in \mathfrak{h}^{*}\right.$ dominant) such that $M \in \mathrm{Ob}\left\langle\mathcal{F}_{0}(\mathfrak{g}) \otimes M^{\prime}\right\rangle$. Suppose $M^{\prime}$ is simple (this is the case, for example, if $M$ is simple or $M$ is a Verma module). By Lemma $3 M^{\prime}$ is non-zero and $\operatorname{Ann}_{U(\mathfrak{g})} M^{\prime}=$ $U(\mathfrak{g})|\mu|^{\prime}$. Moreover it is projective in the category $\left\langle\mathcal{F}(\mathfrak{g}) \otimes M^{\prime}\right\rangle$ (because every translation from the wall and back is a direct sum of some copies of the identity functors, so the category $\left\langle\mathcal{F}(\mathfrak{g}) \otimes M^{\prime}\right\rangle_{\left|\mu^{\prime}\right|}$ is semisimple). The following lemma can be found in [KM2].

Lemma 11. Let $M$ and $M^{\prime}$ be as above. For every $F \in \operatorname{Ob}\left(\mathcal{F}_{0}(\mathfrak{g})\right)$ the canonical map $\operatorname{Hom}_{\mathfrak{g}}\left(F, U(\mathfrak{g}) /\left|\mu^{\prime}\right| U(\mathfrak{g})\right) \rightarrow \operatorname{Hom}_{\mathfrak{g}}\left(F, \mathcal{L}\left(M^{\prime}, M^{\prime}\right)\right)$ given by multiplication is an isomorphism.

Proof. The injectivity follows from the injectivity of canonical morphism $U(\mathfrak{g}) / \operatorname{Ann}_{U(\mathfrak{g})} M^{\prime} \rightarrow \mathcal{L}\left(M^{\prime}, M^{\prime}\right)$ (see [Ja, 6.8]). Let $F$ be a simple object of $\mathcal{F}_{0}(\mathfrak{g})$. By Kostant's theorem describing $U(\mathfrak{g})^{\text {ad }}$ as module over $Z(\mathfrak{g})$ one has $\operatorname{dim} \operatorname{Hom}_{\mathfrak{g}}\left(F,\left(U(\mathfrak{g}) / \operatorname{Ann}_{U(\mathfrak{g})} M^{\prime}\right)^{\text {ad }}\right)=\operatorname{dim} F_{0}$. On the other hand the category $\left\langle\mathcal{F}(\mathfrak{g}) \otimes M^{\prime}\right\rangle_{\left|\mu^{\prime}\right|}$ is semisimple by the construction of $M^{\prime}$ and $\left|\mu^{\prime}\right|$, thus, using Equality (4), we get

$$
\operatorname{Hom}_{\mathfrak{g}}\left(F, \mathcal{L}\left(M^{\prime}, M^{\prime}\right)^{\mathrm{ad}}\right)=\operatorname{Hom}_{\mathfrak{g}}\left(M^{\prime}, F^{*} \otimes M^{\prime}\right)=\operatorname{dim} F_{0}^{*},
$$

and the lemma follows.

Chose $\lambda \in \mathfrak{h}^{*}$ dominant, such that $\operatorname{Ann}_{U(\mathfrak{g})}\left(M^{\prime}\right) M(\lambda)=0$. Applying arguments similar to these in Proposition 3 we get the equivalence of categories

$$
\left.\operatorname{coker}\left(\mathcal{F}_{0}(\mathfrak{g}) \otimes M(\lambda)\right) \cong \operatorname{coker}\left(\mathcal{F}_{0}(\mathfrak{g}) \otimes M^{\prime}\right)\right)
$$


Now we are ready to prove

Theorem 10. Let $M$ be a simple $\mathfrak{g}$-module with a minimal annihilator, then $\mathcal{L}(M, M)$ is a free $U(\mathfrak{g}) / \operatorname{Ann}(M)$-module of rank $\left|\Gamma_{M}\right|$.

Proof. Let $\lambda \in \mathfrak{h}^{*}$ be dominant such that $|\lambda| M=0$. Choose $\gamma \in \Gamma_{M}$. Then $\gamma+\mathbb{Z} R \cap W \cdot \lambda \neq \emptyset$ by the definition of $\Gamma_{M}$. Let $\mu \in \gamma+\mathbb{Z} R \cap$ $W \cdot \lambda$ be dominant. By [BG, Theorem 4.1] the translation functor $T_{\lambda}^{\mu}$ is an equivalence of categories, moreover $M \cong T_{\lambda}^{\mu} M$ (because by construction $\operatorname{Hom}_{\mathfrak{g}}\left(M, T_{\lambda}^{\mu} M\right) \neq 0$ and both modules are simple). This implies

$$
\mathcal{L}(M, M)_{\gamma} \cong \mathcal{L}\left(T_{\lambda}^{\mu} M, M\right)_{\gamma} \cong \mathcal{L}(M, M)_{0}
$$

thus, it is enough to prove that $U(\mathfrak{g}) / \operatorname{Ann}_{U(\mathfrak{g})} M \cong \mathcal{L}(M, M)_{0}$.

By Kostant's theorem and [Ja, 6.8] the morphism

$$
U(\mathfrak{g}) / \operatorname{Ann}_{U(\mathfrak{g})} M \rightarrow \mathcal{L}(M, M)
$$

is injective. We will view $M$ as an object of $M \in \mathrm{Ob}\left\langle\mathcal{F}_{0}(\mathfrak{g}) \otimes M^{\prime}\right\rangle$ for $M^{\prime}$ constructed above. To complete the proof of the theorem we will use the coapproximation functor $\tilde{\mathcal{C}}=\tilde{\mathcal{C}}_{M^{\prime}}:\left\langle\mathcal{F}_{0}(\mathfrak{g}) \otimes M^{\prime}\right\rangle \rightarrow \operatorname{coker}\left(\mathcal{F}_{0}(\mathfrak{g}) \otimes M^{\prime}\right)$ constructed in Section 4.

Let $P$ be the translation of $M^{\prime}$ to the character $|\lambda|$. The module $P$ is projective in $\left\langle\mathcal{F}_{0}(\mathfrak{g}) \otimes M^{\prime}\right\rangle$ and $\operatorname{coker}\left(\mathcal{F}_{0}(\mathfrak{g}) \otimes M^{\prime}\right)_{|\lambda|}$ is the subcategory of all $P$-presentable modules. From the description of endomorphisms of antidominant projective modules in $\mathcal{O}$ (given e.g. in [S90]) and using Equivalence (5), one gets that $\operatorname{End}_{\mathfrak{g}}(P)$ is a local commutative algebra with the maximal ideal, say $\mathfrak{m}$. Since $M$ is simple, $\operatorname{Hom}(P, M)=\mathbb{C}$, thus from the construction of $\tilde{\mathcal{C}}$ it follows that $\tilde{\mathcal{C}}(M)=P / \mathfrak{m} P$, thus under Equivalence $(5)$ the module $\tilde{\mathcal{C}}(M)$ corresponds to the module $P\left(w_{0} \cdot \lambda\right) / P\left(w_{0} \cdot \lambda\right) \mathfrak{m} \cong M(\lambda)^{*}$.

Obviously, for any simple finite dimensional module $F$ one has the isomorphism $\tilde{\mathcal{C}}(F \otimes M) \cong F \otimes \tilde{\mathcal{C}}(M)$. Dualizing [Ja, 6.9(10)] we get $U(\mathfrak{g}) / \operatorname{Ann}_{U(\mathfrak{g})} M(\lambda)^{*} \cong \mathcal{L}\left(M(\lambda)^{*}, M(\lambda)^{*}\right)$. Again using Kostant's theorem like in the proof of Lemma 11 we get

$\operatorname{dim} F_{0}=\operatorname{dim} \operatorname{Hom}_{\mathfrak{g}}\left(M(\mu)^{*}, F \otimes M(\mu)^{*}\right)=\operatorname{dim} \operatorname{Hom}_{\mathfrak{g}}(\tilde{\mathcal{C}}(M), F \otimes \tilde{\mathcal{C}}(M))$.

Since $M$ is simple and $\tilde{\mathcal{C}}(M) \neq 0$ the canonical adjunction morphism $\mathfrak{i} \circ \tilde{\mathcal{C}}(M) \rightarrow M$ is surjective thus by Lemma 5 the canonical map

$$
\operatorname{Hom}_{\mathfrak{g}}(M, F \otimes M) \rightarrow \operatorname{Hom}_{\mathfrak{g}}(\tilde{\mathcal{C}}(M), F \otimes \tilde{\mathcal{C}}(M))
$$

is injective, i.e.

$$
\operatorname{dim} \operatorname{Hom}_{\mathfrak{g}}\left(F, \mathcal{L}(M, M)_{0}\right) \leq \operatorname{dim} \operatorname{Hom}_{\mathfrak{g}}\left(F, \mathcal{L}(\tilde{\mathcal{C}}(M), \tilde{\mathcal{C}}(M))_{0}\right) .
$$

This completes the proof of the theorem. 
Remark 4. The arguments in the proof of Theorem 10 can also be applied to prove that for every dual Verma module $M$ the canonical map $U(\mathfrak{g}) / \operatorname{Ann}_{U(\mathfrak{g})} M \rightarrow \mathcal{L}(M, M)$ is an isomorphism. The dual statement for Verma modules is well known, and a modification of the above arguments provide an elegant proof of it.

Let $\mathfrak{p}$ be a parabolic subalgebra of $\mathfrak{g}$. Theorem 10 (under certain restrictions) can be generalized to modules $M$ such that the annihilator of $M$ is the annihilator of generalized Verma module in parabolic category $\mathcal{O}^{\mathfrak{p}}$ (so called induced ideals). The crucial point of the construction is the existence of simple projective module in $\mathcal{O}$. In parabolic category $\mathcal{O}^{\mathfrak{p}}$ such modules exist for maximal parabolic subalgebra and for an arbitrary parabolic subalgebra of Lie algebra of type $A$, see [IS] for more details.

\section{Structure of Induced Modules}

In this section we present the results of [KM2]. Let $\mathfrak{p}, \mathfrak{m}, \mathfrak{u}$ be as in Section 3. The semisimple part of $\mathfrak{m}$ will be denoted by $\mathfrak{a}$ and let $\mathfrak{h}^{\perp} \subset \mathfrak{m}$ be the orthogonal complement (with respect to Killing form) of $\mathfrak{h} \cap \mathfrak{a}$ in $\mathfrak{h}$. Every $\mathfrak{m}$-module $N$ can be viewed as a $\mathfrak{p}$-module with the trivial action of $\mathfrak{u}$. Let $M_{\mathfrak{p}}(N)$ denote the parabolicaly induced module $U(\mathfrak{g}) \bigotimes_{U(\mathfrak{p})} N$. We say that an m-module $N$ has integral resp. dominant central character if the parabolicaly induced module $M_{\mathfrak{p}}(N)$ has integral central character resp. the action of $\mathfrak{h}^{\perp}$ on $N$ is given by the restriction of some dominant $\lambda \in \mathfrak{h}^{*}$. In this section we consider only ( $\mathfrak{m}$ - or $\mathfrak{g}$-) modules with integral central character, the difficulties in the general case are mostly in introducing enough indexes. A more or less complete treatment can be found in [KM2].

For an a-module, $M$, we define the rough length $\mathrm{RL}(M)$ of $M$ to be the supremum of the length of all filtrations

$$
0=M_{0} \subset M_{1} \subset \cdots \subset M_{n}=M
$$

such that $\operatorname{Ann}_{U(\mathfrak{a})} M_{i} / M_{i-1}$ is contained in a minimal primitive ideal of $U(\mathfrak{a})$ for all $1 \leq i \leq n$ and zero if such filtration does not exist. The same notion can be defined for $\mathfrak{g}$-modules and we will use $\operatorname{RL}_{\mathfrak{g}}(M)$ in this case. It happens that this invariant behaves well under tensoring with finite-dimensional modules.

Lemma 12. Assume that $M$ is an $\mathfrak{a}$-module of finite rough length. Then $\operatorname{RL}(F \otimes M)=\operatorname{dim}(F) \operatorname{RL}(M)$ for every finite dimensional $\mathfrak{a}$ module $F$. 
Proof. Using exactness of the tensor product with $F$ we first reduce the statement to the case $\operatorname{RL}(M)=1$, without loss of generality we can assume that $M$ is a simple module with minimal annihilator. Tensoring with finite dimensional module is a direct sum of irreducible projective functors by $[\mathrm{BG}]$. Each irreducible projective functor is a direct summand of a product of regular translations, translations on and from the wall. Thus it is enough to check the Lemma in all these cases.

The standard properties of the translation functors show that the regular translations (those described in [BG, Theorem 4.1]) and translations to the walls send simples to simples (see e.g. [BeGi, Proposition 3.1]) and thus does not change the rough length. Having this we can translate from the wall and then back to the wall without crossing other walls, which will be a direct sum of some copies of the identity functor. Hence, by Lemma 3, the rough length of the result does not depend on $M$. This means that we can check our statement for example on simple (or projective) Verma modules, for which it is well known.

Let $M$ be a $\mathfrak{g}$ module and $\lambda \in\left(\mathfrak{h}^{\perp}\right)^{*}$. Set

$$
M_{\lambda}=\left\{m \in M: h(m)=\lambda(h) m \text { for all } h \in \mathfrak{h}^{\perp}\right\} .
$$

If $M=\bigoplus_{\lambda \in\left(\mathfrak{h}^{\perp}\right)^{*}} M_{\lambda}$ we define the rough $\mathfrak{a}$-character of $M$ to be the function $\operatorname{ch}_{\mathfrak{a}}^{M}:\left(\mathfrak{h}^{\perp}\right)^{*} \rightarrow \mathbb{Z} \cup\{\infty\}$ such that $\operatorname{ch}_{\mathfrak{a}}(\lambda)=\operatorname{RL}\left(M_{\lambda}\right)$. We note that, by Lemma $12, \operatorname{ch}_{\mathfrak{a}}^{M_{\mathfrak{p}}(N)}(\lambda)<\infty$ for all $\lambda \in \mathfrak{h}_{\mathfrak{a}}^{*}$ if and only if $\operatorname{RL}(N)<\infty$.

Let $\mathfrak{u}_{-}$be the image of $\mathfrak{u} \subset \mathfrak{n}_{+}$under the standard Chevalley involution interchanging $\mathfrak{n}_{+}$and $\mathfrak{n}_{-}$. Similarly to Verma modules, the induced module $M_{\mathfrak{p}}(N)$ has the following universal property: If $M$ is a $\mathfrak{g}$-module such that for some $\lambda \in\left(\mathfrak{h}^{\perp}\right)^{*}$ holds $M_{\lambda} \mid \cong N$ as $\tilde{\mathfrak{a}}$-modules $M$ is generated by $M_{\lambda}$ as $\mathfrak{g}$-module and $\mathfrak{u} M_{\lambda}=0$ then $M$ is a quotient of $M_{\mathfrak{p}}(N)$. Moreover if $M$ is $\mathfrak{u}_{-}$-free then $M \cong M_{\mathfrak{p}}(N)$.

Let $N^{\prime}$ be a simple $\mathfrak{m}$-module with a minimal annihilator (in particular, its arbitrary translation is non-zero by Lemma 3). Let the the action of $\mathfrak{h}^{\perp}$ be given by the restriction of $\mu \in \mathfrak{h}^{*}$. Let $N$ be an $\mathfrak{m}$ module such that as a-module $N$ is the translation of $N^{\prime}$ to the most singular character, $-\rho_{\mathfrak{a}}$, (here $\rho_{\mathfrak{a}}$ denotes half the sum of positive roots of $\mathfrak{a})$ and the action of $\mathfrak{h}^{\perp}$ is given by the restriction of dominant regular $\lambda \in \mathfrak{h}^{*}$ such that $\mu-\lambda \in \mathbb{Z} R$. (In particular $N$ is simple $\mathfrak{a}$-module.) The next lemma describes the most important properties of the induced module $M_{\mathfrak{p}}(N)$.

Lemma 13. Let $N^{\prime}$ and $N$ be as above. 
(1) $M_{\mathfrak{p}}(N)$ is projective in $\left\langle\mathcal{F}(\mathfrak{g}) \otimes M_{\mathfrak{p}}(N)\right\rangle$.

(2) $M_{\mathfrak{p}}\left(N^{\prime}\right) \in \mathrm{Ob}\left\langle\mathcal{F}(\mathfrak{g}) \otimes M_{\mathfrak{p}}(N)\right\rangle$.

(3) $U(\mathfrak{g}) / \operatorname{Ann}_{U(\mathfrak{g})} M_{\mathfrak{p}}(N) \cong \mathcal{L}\left(M_{\mathfrak{p}}(N), M_{\mathfrak{p}}(N)\right)$.

Proof. (1) This follows from the universal property of induced modules and $S$-Harish-Chandra isomorphism [DFO].

(2) By construction $M_{\mathfrak{p}}(N) \in \mathrm{Ob}\left\langle\mathcal{F}(\mathfrak{g}) \otimes M_{\mathfrak{p}}\left(N^{\prime}\right)\right\rangle$. By $S$-HarishChandra homomorphism and universal property of induced modules, there exist a finite dimensional module $E \in \mathcal{F}_{0}(\mathfrak{g})$ such that

$$
\operatorname{Hom}_{\mathfrak{g}}\left(M_{\mathfrak{p}}(N), E \otimes M_{\mathfrak{p}}\left(N^{\prime}\right)\right) \neq 0 .
$$

By adjunction there exist a non-trivial morphism $f: E^{*} \otimes M_{\mathfrak{p}}(N) \rightarrow$ $M_{\mathfrak{p}}\left(N^{\prime}\right)$. It is an epimorphism because both modules are $\mathfrak{n}_{-} \cap \mathfrak{a}$-free and $N^{\prime}$ is simple.

(3) The canonical map $U(\mathfrak{g}) / \operatorname{Ann}_{U(\mathfrak{g})} M_{\mathfrak{p}}(N) \rightarrow \mathcal{L}\left(M_{\mathfrak{p}}(N), M_{\mathfrak{p}}(N)\right)$ given by multiplication is injective by $[\mathrm{Ja}, 6.8]$. Chose simple finite dimensional module $F$. If $F_{0}=0$, then $\operatorname{Hom}_{\mathfrak{g}}\left(M_{\mathfrak{p}}(N), F \otimes M_{\mathfrak{p}}(N)\right)=$ 0 because we restricted ourself to the modules with integral central character. If $F_{0} \neq 0$ then by [Ja][6.8]

$$
\operatorname{dim} \operatorname{Hom}_{\mathfrak{g}}\left(F,\left(\operatorname{End}\left(M_{\mathfrak{p}}(N)\right)^{\operatorname{ad}}\right)\right)=\operatorname{dim} \operatorname{Hom}_{\mathfrak{g}}\left(M_{\mathfrak{p}}(N), F^{*} \otimes M_{\mathfrak{p}}(N)\right)
$$

and using the projectivity of $M_{\mathfrak{p}}(N)$ and the fact that $N$ has the most singular central character as $\mathfrak{a}$-module we get

$$
\operatorname{dim} \operatorname{Hom}_{\mathfrak{g}}\left(M_{\mathfrak{p}}(N), F^{*} \otimes M_{\mathfrak{p}}(N)\right)=\operatorname{dim} F_{0} .
$$

From the other side, by Kostant's theorem

$$
\operatorname{dim} \operatorname{Hom}_{\mathfrak{g}}\left(F,\left(U(\mathfrak{g}) / \operatorname{Ann}_{U}(\mathfrak{g}) M_{\mathfrak{p}}(N)\right)^{\operatorname{ad}}\right)=\operatorname{dim} F_{0}
$$

because $\operatorname{Ann}_{U(\mathfrak{g})} M_{\mathfrak{p}}(N)$ is the minimal primitive ideal (this follows for example from [MS, Lemma 5.9]).

Let $\lambda \in \mathfrak{h}^{*}$ be dominant such that $\operatorname{Ann}_{Z(\mathfrak{g})} M(\lambda)=\operatorname{Ann}_{Z(\mathfrak{g})} M_{\mathfrak{p}}(N)$. The above lemma together with Proposition 3 implies the equivalence of categories

$$
\operatorname{coker}(\mathcal{F}(\mathfrak{g}) \otimes M(\lambda)) \cong \operatorname{coker}\left(\mathcal{F}(\mathfrak{g}) \otimes M_{\mathfrak{p}}(N)\right) .
$$

This equivalence is a key point in [MS, KM2]. Using our technique, it can be established without introducing the category of Harish-Chandra bimodules. Let us show how one can get the information about the structure of induced modules from the above equivalence. First we need the following description of $\operatorname{ker} \tilde{\mathcal{C}}_{M_{\mathfrak{p}}(N)}$.

Lemma 14. Let $M$ be an object of $\left\langle\mathcal{F}(\mathfrak{g}) \otimes M_{\mathfrak{p}}(N)\right\rangle$. Then $\tilde{\mathcal{C}}_{M_{\mathfrak{p}}(N)}(M)=$ 0 if and only if $\mathrm{ch}_{\mathfrak{a}}^{M}=0$. 
Proof. Suppose $\tilde{\mathcal{C}}_{M_{\mathfrak{p}}(N)}(M) \neq 0$, then, restricting to $\mathfrak{a}$, we get that there exist an indecomposable direct summand $\tilde{N}$ of $\left.\tilde{\mathcal{C}}_{M_{\mathfrak{p}}(N)}(M)\right|_{\mathfrak{a}}$, finite dimensional a-modules $E_{1}$ and $E_{2}$ and a morphism $f: E_{1} \otimes N \rightarrow E_{2} \otimes N$ such that $\tilde{N} \cong$ coker $f$. Composing the projection $E_{2} \otimes N \rightarrow \tilde{N}$ with the inclusion $\left.\tilde{N} \rightarrow M\right|_{\mathfrak{a}}$ we get a non-trivial map $p:\left.E_{2} \otimes N \rightarrow M\right|_{\mathfrak{a}}$. By adjunction one gets a non-trivial morphism $p^{\prime}: N \rightarrow E_{2}^{*} \otimes\left(\left.M\right|_{\mathfrak{a}}\right)$. Since $N$ is simple, this map is injective. Since $N$ has non-zero rough character by Lemma 12 we get that $\operatorname{ch}_{\mathfrak{a}}^{M} \neq 0$.

Suppose now that $\operatorname{ch}_{\mathfrak{a}}^{M} \neq 0$. Let $\nu \in\left(\mathfrak{h}^{\perp}\right)^{*}$ be maximal such that $\operatorname{ch}_{\mathfrak{a}}^{M}(\nu) \neq 0$ (maximal with respect to the partial order on $\left(\mathfrak{h}^{\perp}\right)^{*}$ induced from the standard one on $\left.\mathfrak{h}^{*}\right)$. From definition of rough character follows that $M_{\nu}$ contains an a-submodule $\tilde{N}$ with simple top of rough length 1 . The maximality of $\nu$ implies that $\mathfrak{u} \tilde{N}=0$, thus, by the universal property of induced modules, there exist a non-trivial morphism $g: M_{\mathfrak{p}}(\tilde{N}) \rightarrow M$. Now, applying appropriate projective (or even translation) functor $\mathcal{F}$, we can achieve that $\mathcal{F}\left(M_{\mathfrak{p}}(\tilde{N})\right)$ has the same central character as $M_{\mathfrak{p}}(N)$ and $\mathcal{F}\left(M_{\mathfrak{p}}(\tilde{N})\right)_{\left.\lambda\right|_{\mathfrak{h}} \perp} \neq 0$. Recall that $\left.\lambda\right|_{\mathfrak{h}^{\perp}}$ is the highest $\mathfrak{h}^{\perp}$-weight of $M_{\mathfrak{p}}(N)$.

By construction, we get a map $N \rightarrow \mathcal{F}\left(M_{\mathfrak{p}}(\tilde{N})\right)_{\left.\lambda\right|_{\mathfrak{h} \perp}}$ of $\mathfrak{a}$-modules, which can be extended by universal property of induced modules to a map $g: M_{\mathfrak{p}}(N) \rightarrow \mathcal{F}\left(M_{\mathfrak{p}}(\tilde{N})\right)$ (here we use that the action of $\left(\mathfrak{h}^{\perp}\right)^{*}$ on $N$ is given by the restriction of a dominant weight). So, by adjunction, we get a non-trivial map $E \otimes M_{\mathfrak{p}}(N) \rightarrow M_{\mathfrak{p}}(\tilde{N}) \rightarrow M$ for some finite dimensional module $E$. This implies that $\tilde{\mathcal{C}}_{M_{\mathfrak{p}}(N)}(M) \neq 0$.

Now we present the generalizations of some known facts about Verma modules.

Proposition 9. Let $V$ be a simple $\mathfrak{m}$-module, then the socle of $M_{\mathfrak{p}}(V)$ is simple and has non-zero rough character.

Proof. Under the assumption that $V$ has a minimal annihilator, one can adopt the classical growth arguments. By standard arguments it follows from Lemma 12 that the growth of $\operatorname{ch}_{\mathfrak{a}}^{M_{\mathfrak{p}}(V)}$ is polynomial and that all GVMs of the form $M_{\mathfrak{p}}(Y)$, where $Y$ is a simple subquotient with rough length 1 of some $F \otimes V, F \in \mathcal{F}(\tilde{\mathfrak{a}})$, have the same rough a-character up to a shift.

Further we note that the socle of every $F \otimes V$ as above contains only simples of rough length 1 . Indeed, this is trivial for translations through the walls and then this extends to every $F \otimes_{-}$. It follows that each submodule of $M_{\mathfrak{p}}(V)$ contains in its turn a submodule, isomorphic to some $M_{\mathfrak{p}}(Y)$ with $Y$ as above. Since the leading coefficient of the 
growth polynomial for the rough $\mathfrak{a}$-character of $M_{\mathfrak{p}}(V)$ behaves additively with respect to the direct sums, we get that the socle of $M_{\mathfrak{p}}(V)$ can contain only one copy of $M_{\mathfrak{p}}(Y)$ and hence is simple.

Proposition 10. Let $V_{i}, i=1,2$, be two simple $\tilde{\mathfrak{a}}$-modules with minimal annihilators. Then the dimension of $\operatorname{Hom}_{\mathfrak{g}}\left(M_{\mathfrak{p}}\left(V_{1}\right), M_{\mathfrak{p}}\left(V_{2}\right)\right)$ is at most one and every non-zero element of this space is injective.

Proof. We adopt the proof of [Di, Theorem 7.6.6].

(i) The injectivity easily follows from the universal property of induced modules.

(ii) Let $\phi_{1}, \phi_{2} \in \operatorname{Hom}_{\mathfrak{g}}\left(M_{\mathfrak{p}}\left(V_{1}\right), M_{\mathfrak{p}}\left(V_{2}\right)\right)$ be such that $\phi_{1}\left(M_{\mathfrak{p}}\left(V_{1}\right)\right)=$ $\phi_{2}\left(M_{\mathfrak{p}}\left(V_{1}\right)\right)$. From (i) there exist an endomorphism $\alpha$ of $M_{\mathfrak{p}}\left(V_{1}\right)$ such that $\phi_{2}=\phi_{1} \circ \alpha$. Since $V_{1}$ is simple $\alpha$ is scalar, hence $\phi_{1}$ and $\phi_{2}$ are linearly dependent.

(iii) Assume $M_{\mathfrak{p}}\left(V_{1}\right)$ is simple and $\phi_{1}, \phi_{2} \in \operatorname{Hom}_{\mathfrak{g}}\left(M_{\mathfrak{p}}\left(V_{1}\right), M_{\mathfrak{p}}\left(V_{2}\right)\right)$. By Proposition $9 \phi_{1}\left(M_{\mathfrak{p}}\left(V_{1}\right)\right)=\phi_{2}\left(M_{\mathfrak{p}}\left(V_{1}\right)\right)$, thus $\phi_{1}$ and $\phi_{2}$ are linearly dependent.

(iv) By Proposition 9 there exist a simple $\mathfrak{m}$-module $V$ such that $M_{\mathfrak{p}}(V)$ is simple socle of $M_{\mathfrak{p}}\left(V_{1}\right)$. Let $\mathfrak{i}: M_{\mathfrak{p}}(V) \rightarrow M_{\mathfrak{p}}\left(V_{1}\right)$ be the inclusion. By (i), the composition with $\mathfrak{i}$ defines an injective linear map from $\operatorname{Hom}_{\mathfrak{g}}\left(M_{\mathfrak{p}}\left(V_{1}\right), M_{\mathfrak{p}}\left(V_{2}\right)\right)$ to $\operatorname{Hom}_{\mathfrak{g}}\left(M_{\mathfrak{p}}(V), M_{\mathfrak{p}}\left(V_{2}\right)\right)$. The last space is 1-dimensional by (iii). This completes the proof.

By construction, for simple $\mathfrak{m}$-module, $V$, the module $M_{\mathfrak{p}}(V)$ has simple top which will be denoted by $L_{\mathfrak{p}}(V)$. Now we would like to associate a Verma module to the GVM $M_{\mathfrak{p}}(V)$. For this we denote by $\mathfrak{f}(V)$ the Verma module $M(\nu)$ over $\tilde{a}$ such that the induced module $M_{\mathfrak{p}}\left(M_{\nu}\right)$ has the same central character as $V$ and $\mu$ belongs to the closure of the antidominant Weyl chamber (this one is uniquely defined). Then the module $M_{\mathfrak{p}}(M(\nu))=M_{\mathfrak{p}}(\mathfrak{f}(V))$ is Verma module over $\mathfrak{g}$.

Theorem 11. Let $V_{1}$ and $V_{2}$ be two simple $\mathfrak{m}$-modules with minimal annihilator and as $\mathfrak{a}$-modules $V_{1} \in\left\langle\mathcal{F}_{0}(\tilde{\mathfrak{a}}) \otimes V_{2}\right\rangle$. Then $M_{\mathfrak{p}}\left(V_{2}\right)$ is a submodule of $M_{\mathfrak{p}}\left(V_{1}\right)$ if and only if $M_{\mathfrak{p}}\left(\mathfrak{f}\left(V_{2}\right)\right)$ is a submodule of $M_{\mathfrak{p}}\left(\mathfrak{f}\left(V_{1}\right)\right)$, moreover

$$
\left[M_{\mathfrak{p}}\left(V_{1}\right): L_{\mathfrak{p}}\left(V_{2}\right)\right]=\left[M_{\mathfrak{p}}\left(\mathfrak{f}\left(V_{1}\right)\right): L_{\mathfrak{p}}\left(\mathfrak{f}\left(V_{2}\right)\right)\right] .
$$

Proof. Like in Lemma 13 we can find a m-module $N$ with minimal annihilator, such that $M_{\mathfrak{p}}(N)$ is projective in $\left\langle\mathcal{F}(\mathfrak{g}) \otimes M_{\mathfrak{p}}(N)\right\rangle$ and $M_{\mathfrak{p}}\left(V_{1}\right), M_{\mathfrak{p}}\left(V_{2}\right) \in \mathrm{Ob}\left\langle\mathcal{F}(\mathfrak{g}) \otimes M_{\mathfrak{p}}(N)\right\rangle$.

By Lemma $14 \tilde{\mathcal{C}}_{M_{\mathfrak{p}}(N)}\left(M_{\mathfrak{p}}\left(V_{1}\right)\right) \neq 0$ and $\tilde{\mathcal{C}}_{M_{\mathfrak{p}}(N)}\left(L_{\mathfrak{p}}\left(V_{2}\right)\right) \neq 0$. From the simplicity of $V_{1}$ it follows that the canonical adjunction morphisms 
$\mathfrak{i} \circ \tilde{\mathcal{C}}_{M_{\mathfrak{p}}(N)}\left(M_{\mathfrak{p}}\left(V_{2}\right)\right) \rightarrow M_{\mathfrak{p}}\left(V_{2}\right)$ and $\mathfrak{i} \circ \tilde{\mathcal{C}}_{M_{\mathfrak{p}}(N)}\left(L_{\mathfrak{p}}\left(V_{2}\right)\right) \rightarrow L_{\mathfrak{p}}\left(V_{2}\right)$ are surjective, thus, by Lemma 5 , the canonical maps

$$
\operatorname{Hom}_{\mathfrak{g}}\left(M_{\mathfrak{p}}\left(V_{2}\right), M_{\mathfrak{p}}\left(V_{1}\right)\right) \rightarrow \operatorname{Hom}_{\mathfrak{g}}\left(\tilde{\mathcal{C}}_{M_{\mathfrak{p}}(N)}\left(M_{\mathfrak{p}}\left(V_{2}\right)\right), \tilde{\mathcal{C}}_{M_{\mathfrak{p}}(N)}\left(M_{\mathfrak{p}}\left(V_{1}\right)\right)\right)
$$

and

(7) $\operatorname{Hom}_{\mathfrak{g}}\left(L_{\mathfrak{p}}\left(V_{2}\right), M_{\mathfrak{p}}\left(V_{1}\right)\right) \rightarrow \operatorname{Hom}_{\mathfrak{g}}\left(\tilde{\mathcal{C}}_{M_{\mathfrak{p}}(N)}\left(L_{\mathfrak{p}}\left(V_{2}\right)\right), \tilde{\mathcal{C}}_{M_{\mathfrak{p}}(N)}\left(M_{\mathfrak{p}}\left(V_{1}\right)\right)\right)$

are injective. The bijectivity of the first map follows from the next Lemma.

Lemma 15. There is an injective map

$$
\operatorname{Hom}_{\mathfrak{g}}\left(\tilde{\mathcal{C}}_{M_{\mathfrak{p}}(N)}\left(M_{\mathfrak{p}}\left(V_{2}\right)\right), \tilde{\mathcal{C}}_{M_{\mathfrak{p}}(N)}\left(M_{\mathfrak{p}}\left(V_{1}\right)\right)\right) \hookrightarrow \operatorname{Hom}_{\mathfrak{g}}\left(M_{\mathfrak{p}}\left(V_{2}\right), M_{\mathfrak{p}}\left(V_{1}\right)\right) .
$$

Proof of the lemma. We start with two exact sequences:

$$
\begin{aligned}
0 & \rightarrow N_{1} \rightarrow \tilde{\mathcal{C}}_{M_{\mathfrak{p}}(N)}\left(M_{\mathfrak{p}}\left(V_{1}\right)\right) \rightarrow M_{\mathfrak{p}}\left(V_{1}\right) \rightarrow 0, \\
0 & \rightarrow N_{2} \rightarrow \tilde{\mathcal{C}}_{M_{\mathfrak{p}}(N)}\left(M_{\mathfrak{p}}\left(V_{2}\right)\right) \rightarrow M_{\mathfrak{p}}\left(V_{2}\right) \rightarrow 0,
\end{aligned}
$$

where $\operatorname{ch}_{\mathfrak{a}}^{N_{1}}=\operatorname{ch}_{\mathfrak{a}}^{N_{2}}=0$. Applying $\operatorname{Hom}_{\mathfrak{g}}\left(-, M_{\mathfrak{p}}\left(V_{1}\right)\right)$ to (9) we get the exact sequence

$$
\begin{aligned}
& 0 \rightarrow \operatorname{Hom}_{\mathfrak{g}}(\left.M_{\mathfrak{p}}\left(V_{2}\right), M_{\mathfrak{p}}\left(V_{1}\right)\right) \rightarrow \\
& \operatorname{Hom}_{\mathfrak{g}}\left(\tilde{\mathcal{C}}_{M_{\mathfrak{p}}(N)}\left(M_{\mathfrak{p}}\left(V_{2}\right)\right), M_{\mathfrak{p}}\left(V_{1}\right)\right) \rightarrow \operatorname{Hom}_{\mathfrak{g}}\left(N_{2}, M_{\mathfrak{p}}\left(V_{1}\right)\right) .
\end{aligned}
$$

Here $\operatorname{Hom}_{\mathfrak{g}}\left(N_{2}, M_{\mathfrak{p}}\left(V_{1}\right)\right)=0$ since $\mathrm{ch}_{\mathfrak{a}}^{N_{2}}=0$ while the socle of $M_{\mathfrak{p}}\left(V_{1}\right)$ consists only of modules with non-zero rough character by Proposition 9. Hence we get the isomorphism

$$
\operatorname{Hom}_{\mathfrak{g}}\left(M_{\mathfrak{p}}\left(V_{2}\right), M_{\mathfrak{p}}\left(V_{1}\right)\right) \cong \operatorname{Hom}_{\mathfrak{g}}\left(\tilde{\mathcal{C}}_{M_{\mathfrak{p}}(N)}\left(M_{\mathfrak{p}}\left(V_{2}\right)\right), M_{\mathfrak{p}}\left(V_{1}\right)\right) .
$$

Now we apply $\operatorname{Hom}_{\mathfrak{g}}\left(\tilde{\mathcal{C}}_{M_{\mathfrak{p}}(N)}\left(M_{\mathfrak{p}}\left(V_{2}\right)\right),{ }_{-}\right)$to $(8)$ and get

$$
\begin{aligned}
& 0 \rightarrow \operatorname{Hom}_{\mathfrak{g}}\left(\tilde{\mathcal{C}}_{M_{\mathfrak{p}}(N)}\left(M_{\mathfrak{p}}\left(V_{2}\right)\right), N_{1}\right) \rightarrow \\
& \operatorname{Hom}_{\mathfrak{g}}\left(\tilde{\mathcal{C}}_{M_{\mathfrak{p}}(N)}\left(M_{\mathfrak{p}}\left(V_{2}\right)\right), \tilde{\mathcal{C}}_{M_{\mathfrak{p}}(N)}\left(M_{\mathfrak{p}}\left(V_{1}\right)\right)\right) \rightarrow \\
& \operatorname{Hom}_{\mathfrak{g}}\left(\tilde{\mathcal{C}}_{M_{\mathfrak{p}}(N)}\left(M_{\mathfrak{p}}\left(V_{2}\right)\right), M_{\mathfrak{p}}\left(V_{1}\right)\right),
\end{aligned}
$$

Where $\operatorname{Hom}_{\mathfrak{g}}\left(\tilde{\mathcal{C}}_{M_{\mathfrak{p}}(N)}\left(M_{\mathfrak{p}}\left(V_{2}\right)\right), N_{1}\right)=0$ by the same arguments as above applied to the top of $\tilde{\mathcal{C}}_{M_{\mathfrak{p}}(N)}\left(M_{\mathfrak{p}}\left(V_{2}\right)\right)$. Combining these two results we get the required injection.

Proposition 9 implies also that the map in (7) is an isomorphism. Now we can find a finite filtration

$$
0=M_{0} \subset M_{1} \subset \cdots \subset M_{n}=M_{\mathfrak{p}}\left(V_{1}\right)
$$


such that $M_{\mathfrak{p}}\left(V_{1}\right) / M_{i}$ has only simples with non-zero rough character in the socle for $0 \leq i<n$. The exactness of $\tilde{\mathcal{C}}_{M_{\mathfrak{p}}(N)}$ and easy inductive arguments imply

$$
\left[M_{\mathfrak{p}}\left(V_{1}\right): L_{\mathfrak{p}}\left(V_{2}\right)\right]=\left[\tilde{\mathcal{C}}_{M_{\mathfrak{p}}(N)}\left(M_{\mathfrak{p}}\left(V_{1}\right)\right): \tilde{\mathcal{C}}_{M_{\mathfrak{p}}(N)}\left(L_{\mathfrak{p}}\left(V_{2}\right)\right)\right]
$$

Similarly

$$
\left[M_{\mathfrak{p}}\left(\mathfrak{f}\left(V_{1}\right)\right): L_{\mathfrak{p}}\left(\mathfrak{f}\left(V_{2}\right)\right)\right]=\left[\tilde{\mathcal{C}}_{M(\lambda)}\left(M_{\mathfrak{p}}\left(\mathfrak{f}\left(V_{1}\right)\right)\right): \tilde{\mathcal{C}}_{M(\lambda)}\left(L_{\mathfrak{p}}\left(\mathfrak{f}\left(V_{2}\right)\right)\right] .\right.
$$

The theorem follows now from an observation, that under Equivalence $(6)$ the objects $\tilde{\mathcal{C}}_{M_{\mathfrak{p}}(N)}\left(M_{\mathfrak{p}}\left(V_{1}\right)\right)$ correspond to $\tilde{\mathcal{C}}_{M(\lambda)}\left(M_{\mathfrak{p}}\left(\mathfrak{f}\left(V_{1}\right)\right)\right)$ for $i=1,2$ and $\tilde{\mathcal{C}}_{M_{\mathfrak{p}}(N)}\left(L_{\mathfrak{p}}\left(V_{2}\right)\right)$ corresponds to $\tilde{\mathcal{C}}_{M(\lambda)}\left(L_{\mathfrak{p}}\left(\mathfrak{f}\left(V_{2}\right)\right)\right.$ (first because both $\tilde{\mathcal{C}}_{M_{\mathfrak{p}}(N)}\left(M_{\mathfrak{p}}\left(V_{1}\right)\right)$ and $\tilde{\mathcal{C}}_{M(\lambda)}\left(M_{\mathfrak{p}}\left(\mathfrak{f}\left(V_{1}\right)\right)\right)$ are $\mathfrak{u}_{\text {- }}$-free modules with the same highest $\mathfrak{h}^{\perp}$-weight, second because both object are the quotients of corresponding objects by maximal submodules which do not intersect the highest $\mathfrak{h}^{\perp}$-weight space).

The above theorem and Proposition 9 imply that the module induced from a simple module with minimal annihilator is simple if and only if the associated Verma module is simple. We have to remark that even in the case of category $\mathcal{O}$ Theorem 11 does not give complete information about the submodule structure of induced module. Using this theorem one can only trace those submodules (or subquotients) which have non-zero rough character (they do not lie in the kernel of completion functor).

Proposition 11. If $V$ is a simple $\mathfrak{m}$-module with minimal annihilator such that for every finite dimensional module $E$ the length of $E \otimes V$ is equal to $\operatorname{dim} F$ then every subquotient of $M_{\mathfrak{p}}(V)$ has non-zero rough character.

Proof. Restricted to $\mathfrak{m}$, the module $M_{\mathfrak{p}}(V)$ is a direct sum of modules of type $E \otimes V$ for finite dimensional modules $E$, thus every subquotient of $\left.M_{\mathfrak{p}}(V)\right|_{\mathfrak{m}}$ decomposes into a direct sum of modules that are subquotients of $E \otimes V$ for finite dimensional $E$. By assumption and Lemma 12 for every finite dimensional module $E$ the length of $E \otimes V$ is equal to $\operatorname{dim} E=R L(E \otimes V)$ this implies that every non-zero subquotient of $E \otimes V$ has non-zero rough length, thus every non-zero subquotient of $M_{\mathfrak{p}}(V)$ has non-zero rough character.

Under assumptions of the above proposition the kernel of coapproximation functor is zero and Theorem 11 provides a complete description of the structure of induced modules. In particular this generalizes the results of D. Miličić and W. Soergel [MS] for Whittaker modules, the results of $\mathrm{V}$. Mazorchuk and S. Ovsienko [MO] for modules induced 
from generic Gelfand-Zetlin modules and the results of V. Mazorchuk and the author [KM3, KM4] for the modules induced from dense $\operatorname{sl}(2)$ modules.

The general situation is rather complicated, because the tensor product of simple module with finite dimensional one can have infinite length (see [St] for an $\operatorname{sl}(2) \times \operatorname{sl}(2)$ example) and it is not clear (at least to the author) how to trace those subquotients.

\section{REFERENCES}

[An] H.H. Andersen, Twisted Verma modules and their quantized analogues, Contemp. Math. 325, 1-10 (2003)

[AnL] H.H. Andersen, N. Lauritzen, Twisted Verma modules,pp. 1-26. Studies in Memory of Issai Schur, Progress in Math. 210, Birkhäuser (2002)

[AnS] H.H. Andersen, C. Stroppel, Twisting functors on O. Preprint 2003.

[Ar] S. Arkhipov, Algebraic construction of contragradient Verma modules in positive caracteristic. Preprint MPI 1998.

[Au] M. Auslander, Representation theory of Artin algebras. I. Comm. Algebra 1 (1974), 177-268.

[Bc] E. Backelin, The Hom-spaces between projective functors. Represent. Theory 5 (2001), 267-283 (electronic).

[Ba] H. Bass, Algebraic $K$-theory. W. A. Benjamin, Inc., New York-Amsterdam $1968762 \mathrm{pp}$.

[BeGi] A. Beilinson, V. Ginzburg, Wall-crossing functors and $\mathcal{D}$-modules. Represent. Theory 3 (1999), 1-31.

[BG] J.N. Bernstein, S.I. Gelfand, Tensor products of finite- and infinitedimensional representations of semisimple Lie algebras. Compositio Math. 41 (1980), no. 2, 245-285.

[Di] J. Dixmier, Algebres enveloppantes, Gauthier-Villars, Paris, 1974.

[DFO] Yu. A. Drozd, V.M. Futorny, S.A. Ovsienko, S-homomorphism of HarishChandra and $\mathfrak{G}$-modules generated by semiprimitive elements, Ukrainian Math. J. 42 (1990), 1032-1037.

[GJ] O. Gaber, A. Joseph, On the Bernshtein-Gelfand-Gelfand resolution and the Duflo sum formula. Compositio Math. 43 (1981), no. 1, 107-131.

[Ga] P. Gabriel, Des catégories abéliennes, Bull. Soc. Math. France 90 (1962), 323-448.

[IS] R. Irving, B. Shelton, Loewy series and simple projective modules in the category $\mathcal{O}_{S}$. Pacific J. Math. 132 (1988), no. 2, 319-342.

[Ja] J.C. Jantzen, Einhüllende Algebren halbeinfacher Lie-Algebren. Ergebnisse der Mathematik und ihrer Grenzgebiete (3), 3. Springer-Verlag, Berlin, 1983. 298 pp.

[J82] A. Joseph, The Enright functor on the Bernstein-Gelfand-Gelfand category $\mathcal{O}$. Invent. Math. 67 (1982), no. 3, 423-445.

[J83] A. Joseph, Completion functors in the $\mathcal{O}$ category. Noncommutative harmonic analysis and Lie groups (Marseille, 1982), 80-106, Lecture Notes in Math., 1020, Springer, Berlin, 1983.

[KM1] O. Khomenko, V. Mazorchuk, On Arkhipov's and Enright's functors. Preprint 2003. 
[KM2] O. Khomenko, V. Mazorchuk, Structure of modules induced from simple modules with minimal annihilator. Preprint 2002. To appear in Can. J. Math.

[KM3] O. Khomenko, V. Mazorchuk, Generalized Verma modules induced from $\operatorname{sl}(2, \mathbb{C})$ and associated Verma modules. J. Algebra 242 (2001), 561-576.

[KM4] O. Khomenko, V. Mazorchuk, On multiplicities of simple subquotients in generalized Verma modules. Czechoslovak Math. J. 52(127) (2002), 337-343.

[KV] A.W. Knapp, D.A. Vogan, Cohomological Induction and Unitary Representations, Princeton University Press, 1995.

[MO] V. Mazorchuk, S. Ovsienko, Submodule structure of generalized Verma modules induced from generic Gelfand-Zetlin modules. Algebr. Represent. Theory 1 (1998), no. 1, 3-26.

[MS] D. Miličić, W. Soergel, The composition series of modules induced from Whittaker modules. Comment. Math. Helv. 72 (1997), no. 4, 503-520.

[S86] W. Soergel, Équivalences de certaines catégories de g-modules, C.R.Acad.Sc.Paris, t.303, Série I, p. 725-728. (1986)

[S89] W. Soergel, Universelle versus relative Einhüllende: Eine geometrische Untersuchung von Quotienten von universellen Einhüllenden halbeinfacher LieAlgebren, Math. Ann. 284, 177-198 (1989)

[S90] W. Soergel, Kategorie $\mathcal{O}$, perverse Garben und Moduln über den Koinvarianten zur Weylgruppe. J. Amer. Math. Soc. 3 (1990), no. 2, 421-445.

[S92] W. Soergel, The combinatorics of Harish-Chandra bimodules. J. Reine Angew. Math. 429 (1992), 49-74.

[S97] W. Soergel, Charakterformeln für Kipp-Moduln über Kac-Moody-Algebren. Represent. Theory 1 (1997), 115-132 (electronic).

[St] J.T. Stafford, Nonholonomic modules over Weyl algebras and enveloping algebras. Invent. Math. 79 (1985), no. 3, 619-638.

[Za] A. Zahid, Les endomorphismes k-finis des modules de Whittaker. Bull. Soc. Math. France 117 (1989), no. 4, 451-477. 